\title{
STRONG COMPETITION VERSUS FRACTIONAL DIFFUSION: THE CASE OF LOTKA-VOLTERRA INTERACTION
}

\author{
GIANMARIA VERZINI AND ALESSANDRO ZILIO
}

\begin{abstract}
We consider a system of differential equations with nonlinear Steklov boundary conditions, related to the fractional problem

$$
(-\Delta)^{s} \mathbf{u}=\mathbf{f}(x, \mathbf{u})-\beta u_{i}^{p} \sum_{j \neq i} a_{i j} u_{j}^{p},
$$

where $\mathbf{u}=\left(u_{1}, \ldots, u_{k}\right), s \in(0,1), p>0, a_{i j}>0$ and $\beta>0$. When $k=2$ we develop a quasi-optimal regularity theory in $\mathcal{C}^{0, \alpha}$, uniformly w.r.t. $\beta$, for every $\alpha<\alpha_{\mathrm{opt}}=\min (1,2 s)$; moreover we show that the traces of the limiting profiles as $\beta \rightarrow+\infty$ are Lipschitz continuous and segregated. Such results are extended to the case of $k \geq 3$ densities, with some restrictions on $s, p$ and $a_{i j}$.
\end{abstract}

\section{INTRODUCTION}

Let us consider the following stationary differential system, involving $k \geq 2$ non negative densities $u_{i}$ which are subject to diffusion, reaction and competition

$$
(-\Delta)^{s} u_{i}=f_{i}\left(x, u_{i}\right)-\beta u_{i}^{p} \sum_{j \neq i} a_{i j} u_{j}^{q}
$$

settled in $H^{s}\left(\mathbb{R}^{N}\right), N \geq 1$, or in a bounded domain with suitable boundary conditions. In this system, different ranges of the parameter $s$ allow to model the brownian diffusion $(s=1)$, as well as the fractional one $(0<s<1)$, which arises whenever the underlying Gaussian process is replaced by the Levy one, in order to allow discontinuous random walks. In the latter case, the nonlocal operator

$$
(-\Delta)^{s} u(x)=c_{N, s} \mathrm{pv} \int_{\mathbb{R}^{N}} \frac{u(x)-u(\xi)}{|x-\xi|^{N+2 s}} \mathrm{~d} \xi
$$

denotes the $s$-power of the laplacian. Furthermore, the competitive nature of the interaction is driven by the positivity of the parameters $\beta, p, q$ and $a_{i j}, 1 \leq i, j \leq k$. Among others, two types of competition are particularly relevant in the applications:

- the case $p=q=1$, that is the Lotka-Volterra type competition, which is widely used in population dynamics and ecology;

- the case $p=1, q=2$ (and $a_{i j}=a_{j i}$ ), which turns (1.1) into the GrossPitaevskii system: this system arises in the search of solitary waves associated to the cubic Schrödinger system, which is commonly accepted as a model for Bose-Einstein condensation in multiple states, and often used

2010 Mathematics Subject Classification. Primary: 35J65; secondary: 35B40 35R11 92D25.

Key words and phrases. Spatial segregation, monotonicity formulae, blow-up analysis, optimal regularity of limiting profiles, singular perturbations.

Work partially supported by the PRIN2009 grant "Critical Point Theory and Perturbative Methods for Nonlinear Differential Equations". 
also in nonlinear optics. In great contrast with the Lotka-Volterra one, this system has a variational structure.

In the study of (1.1), a peculiar issue is the analysis of the behavior of the densities in the case of strong competition, i.e. when $\beta \rightarrow+\infty$. In such situation, one expects the formation of self-organized patterns, in which the limiting densities are spatially segregated, and the natural questions regard $a$ ) the common regularity shared by families of solutions, uniformly in $\beta$ and $b$ ) the properties of the limiting segregated profile. In facing such questions, typical tools are the blow-up technique and the monotonicity formulae of Alt-Caffarelli-Friedman and of Almgren type.

After [11, 12, 13, the case $s=1$ of standard diffusion has been extensively studied in the last decade. In particular it is known that, both in the case of Lotka-Volterra competition [14, 5] and in the variational one [6, 21, each family of solutions which share a common uniform bounds in the $L^{\infty}$ norm is precompact in the topology of $H^{1} \cap \mathcal{C}^{0, \alpha}$ for every $\alpha<1$; we highlight that this result is quasioptimal, in the sense that $\alpha=1$ is the maximal common regularity allowed for this problem. Furthermore, the limiting profiles (as $\beta \rightarrow+\infty$ ) are solutions of the segregated system

$$
u_{i}\left(-\Delta u_{i}-f_{i}\left(x, u_{i}\right)\right)=0, \quad u_{i} u_{j}=0 \text { for } j \neq i,
$$

they are Lipschitz continuous, and they obey to a weak reflection law which roughly says that, on the free boundary separating two components, the corresponding gradients are equal in magnitude (up to suitable scaling factors depending on the matrix $\left(a_{i j}\right)$ ) and opposite in direction [25. Remarkably, such law is the same for both types of competition [17]. For some related results, in the case of standard diffusion, we also refer to [16, 22. and references therein.

Coming to the anomalous diffusion case $s \in(0,1)$, for the moment only the competition of Gross-Pitaevskii type has been considered in the literature. In such framework, the results above were recently generalized [26, 27, 28] in the following sense: $L^{\infty}$ uniform bounds imply uniform bounds in $H^{s} \cap \mathcal{C}^{0, \alpha}$ (for a suitable extension problem), for every $\alpha<\alpha_{\mathrm{opt}}^{\mathrm{GP}}(s)$. Here the optimal exponent

$$
\alpha_{\mathrm{opt}}^{\mathrm{GP}}(s)=s,
$$

at least when $0<s \leq 1 / 2$; for $1 / 2<s<1$ we could only show that $\alpha_{\mathrm{opt}}^{\mathrm{GP}}(s) \geq 2 s-1$, because of the lack of a clean-up lemma appropriate to exclude self-segregation; see 27 for further details. In any case, this result agrees with the one holding for the standard Laplace operator, since $\alpha_{\mathrm{opt}}^{\mathrm{GP}}(1)=1$. Moreover the limiting profiles satisfy a natural extension to the fractional setting of the system 1.2 , that is

$$
u_{i}\left((-\Delta)^{s} u_{i}-f_{i}\left(x, u_{i}\right)\right)=0, \quad u_{i} u_{j}=0 \text { for } j \neq i,
$$

and the validity of an Almgren monotonicity formula across the free boundary ensures a reflection property, as in the case $s=1$.

Under the perspective just described, in this paper we address the study of system (1.1) in the case $s \in(0,1)$ and

$$
p=q>0 .
$$

We remark that such range of parameters not only includes the Lotka-Volterra competition, but it is of interest also in the complementary case $p \neq 1$. Indeed, in the case of $k=2$ components, such competition appears in the modeling of diffusion flames [7, while in the general case the change of variables $U_{i}=u_{i}^{p}$ turns 
system (1.1) into the one for competing densities subject to fast fractional diffusion (when $p>1$ ), or to fractional diffusion in a porous medium (when $p<1$ ) [18, 3].

As in 26, 27, we state our results for a localized extension problem [10] related to the nonlocal system 1.1, namely the problem

$(P)_{\beta} \quad \begin{cases}L_{a} v_{i}=0 & \text { in } B_{1}^{+} \\ \partial_{\nu}^{a} v_{i}=f_{i, \beta}\left(x, v_{1}, \ldots, v_{k}\right)-\beta v_{i}^{p} \sum_{j \neq i} a_{i j} v_{j}^{p} & \text { on } \partial^{0} B_{1}^{+},\end{cases}$

where we adopt the standard notations $\mathbb{R}_{+}^{N+1}:=\left\{X=(x, y) \in \mathbb{R}^{N} \times \mathbb{R}: y>0\right\}$, $B_{r}^{+}:=B_{r} \cap\{y>0\} \subset \mathbb{R}_{+}^{N+1}, \partial^{+} B_{r}^{+}:=\partial B_{r} \cap\{y>0\}, \partial^{0} B_{r}^{+}:=B_{r} \cap\{y=0\} \subset \mathbb{R}^{N}$, and

$$
L_{a} v:=-\operatorname{div}\left(|y|^{a} \nabla v\right), \quad \partial_{\nu}^{a} v:=\lim _{y \rightarrow 0^{+}}-y^{a} \partial_{y} v,
$$

for $a:=1-2 s \in(-1,1)$. This last condition insures that the weight $y^{a}$ belongs to the Muckenhoupt $A_{2}$-class [23, so that a weak version of $(P)_{\beta}$ can be formulated in the Hilbert space

$$
H^{1 ; a}\left(B_{1}^{+}\right):=\left\{v: \int_{B_{1}^{+}} y^{a}\left(|v|^{2}+|\nabla v|^{2}\right) \mathrm{d} x \mathrm{~d} y<\infty\right\} .
$$

Our first main results concern the full quasi-optimal theory in the case of two densities.

Theorem 1.1. Let $p>0, a_{i j}>0$ for any $j \neq i$, and the reaction terms $f_{i, \beta}$ be continuous and map bounded sets into bounded sets, uniformly w.r.t. $\beta>0$.

If $k=2$ then, for every

$$
\alpha<\alpha_{\mathrm{opt}}(s)=\alpha_{\mathrm{opt}}^{\mathrm{LV}}(s):=\min (2 s, 1)
$$

and $\bar{m}>0$, there exists a constant $C=C(\alpha, \bar{m})$ independent of $\beta$ such that

$$
\left\|\mathbf{v}_{\beta}\right\|_{L^{\infty}\left(B^{+}\right)} \leq \bar{m} \quad \Longrightarrow \quad\left\|\mathbf{v}_{\beta}\right\|_{\mathcal{C}^{0, \alpha}\left(\overline{B_{1 / 2}^{+}}\right)} \leq C,
$$

for every $\mathbf{v}_{\beta}=\left(v_{1, \beta}, v_{2, \beta}\right)$ nonnegative solution of problem $(P)_{\beta}$.

Furthermore, any sequence of uniformly bounded, nonnegative solutions $\left\{\left(v_{1, \beta_{n}}, v_{2, \beta_{n}}\right)\right\}_{n}$, with $\beta_{n} \rightarrow \infty$, converges (up to subsequences) in $\left(H^{1 ; a} \cap \mathcal{C}^{0, \alpha}\right)\left(\overline{B_{1 / 2}^{+}}\right)$to a limiting profile $\left(v_{1}, v_{2}\right)$.

Theorem 1.2. Under the assumption of the previous theorem, let furthermore $f_{i, \beta} \rightarrow f_{i}$ as $\beta \rightarrow \infty$, uniformly on compact sets, with $f_{i}$ Lipschitz continuous. For any limiting profile $\left(v_{1}, v_{2}\right)$ :

- $v_{1}(x, 0), v_{2}(x, 0)$ are Lipschitz continuous (optimal regularity of the traces);

- $v_{1}(x, 0) \cdot v_{2}(x, 0)=0$ (boundary segregation condition);

- $L_{a} v_{1}=L_{a} v_{2}=0$ for $y>0$;

- $\partial_{\nu}^{a}\left(a_{21} v_{1}-a_{12} v_{2}\right)=a_{21} f_{1}-a_{12} f_{2}$ for $y=0$.

Remark 1.3. In the previous results $B_{1 / 2}^{+}$can be replaced by any domain $\Omega \cap\{y>$ $0\}$, where $\bar{\Omega} \subset B_{1}$.

Remark 1.4. Throughout this paper, we restrict our discussion to nonnegative solutions only to avoid technicalities. Reasoning as in [26], also changing sign solutions can be considered, once the competition is suitably extended to negative densities. 

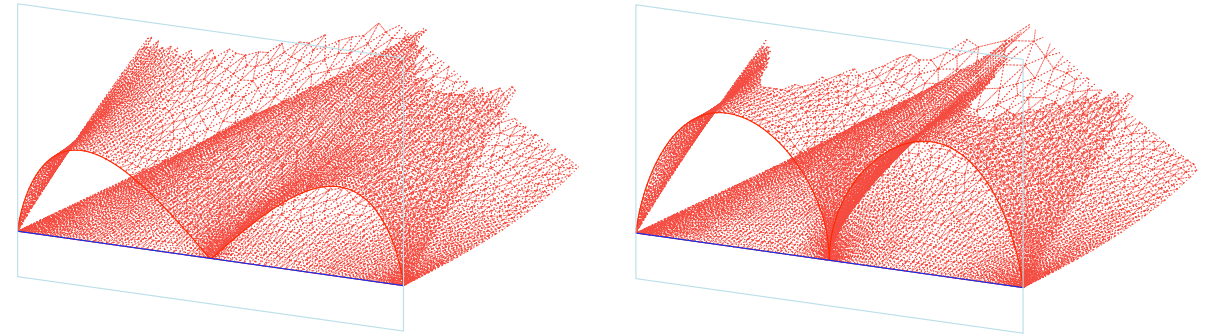

Figure 1. On the left, a numerical approximation of a limiting profile for problem $(P)_{\beta}$ with Lotka-Volterra competition $p=q=$ 1 and $s=1 / 2$, for which Lipschitz continuity of the segregated traces is shown in Theorem 1.2. On the right, the simulation for the analogous problem with Gross-Pitaevskii competition $p=1$, $q=2$, which optimal regularity, according to [26, Theorem 1.2], is only $\mathcal{C}^{0,1 / 2}$.

Remark 1.5. The upper bound $\alpha=2 s$ for the regularity of the functions $v_{i, \beta}$ can not be removed: indeed, from any solution of $(P)_{\beta}$ we can construct another solution having $(k+1)$ components, by defining $v_{k+1, \beta}(x, y)=y^{2 s}, f_{k+1, \beta} \equiv-2 s$. One may possibly expect to be able to remove such threshold by considering only the regularity of the traces $v_{i, \beta}(x, 0)$, as suggested by Theorem 1.2 .

On the other hand, the Lipschitz regularity is the natural one, at least for the traces, since the last condition in Theorem 1.2 implies that $v_{i}(x, 0)$ are (proportional to) the positive/negative parts of a regular function.

Next, we address the case of $k \geq 3$ densities.

Theorem 1.6. Let $k \geq 3$. Then there exists $\alpha^{*}>0$ such that Theorem 1.1 holds for any $\alpha<\alpha^{*}$, under the further assumption that

$$
\text { either } p \geq 1 \quad \text { or } \quad a_{i j}=1 \text { for every } j \neq i .
$$

Furthermore, if $a_{i j}=1$,

$$
\alpha^{*}=\alpha_{\mathrm{opt}}(s)=\min (2 s, 1)
$$

whenever $s=1 / 2$ or $s \in(0,1 / 4)$.

Even though we can show quasi-optimality only in some cases, the above regularity result is sufficient to conclude that, as $\beta \rightarrow \infty$, solutions of $(P)_{\beta}$ accumulate to limiting profiles $v_{i}$ which properties, apart from optimal regularity, are analogous to those described in Theorem 1.2 for the case $k=2$ (see Section 5 for further details). In particular, going back to the segregated traces $u_{i}(x)=v_{i}(x, 0)$, we can show that

$$
u_{i}\left[(-\Delta)^{s}\left(u_{i}-\sum_{j \neq i} \frac{a_{i j}}{a_{j i}} u_{j}\right)-\left(f_{i}-\sum_{j \neq i} \frac{a_{i j}}{a_{j i}} f_{j}\right)\right]=0, \quad u_{i} u_{j}=0 \text { for } j \neq i .
$$

Comparing with equation 1.3 , we see that, if $s<1$, the Gross-Pitaevskii competition and the Lotka-Volterra one exhibit deep differences not only from the point of view of the optimal regularity exponent, but also from that of the differential equations satisfied by the segregated limiting profiles. This is in great contrast with 
the case $s=1$ where, as we already mentioned, the two competitions can not be distinguished from each other in the limit. Such feature is caused by the non local nature of the diffusion operators: indeed equation (1.4) can not be directly reduced to 1.3 , since in the set $\left\{u_{i}=0\right\}$ the corresponding fractional laplacian does not necessarily vanish. Nonetheless, letting $s \rightarrow 1^{-}$, we recover the local nature of the equation: as a consequence

$$
u_{i}(-\Delta)^{s}\left(u_{i}-\sum_{j \neq i} \frac{a_{i j}}{a_{j i}} u_{j}\right) \rightarrow u_{i}\left(-\Delta u_{i}\right)
$$

so that equation 1.2 arises also in this case.

To conclude, we mention that the equations just discussed -or, better, the corresponding ones for the extensions $v_{i}$ - can be used to obtain further regularity for the limiting profiles, also in the case $a_{i j} \neq 1$. In particular, we have the following result.

Theorem 1.7. Let $k \geq 3, s=1 / 2, p>1$. If furthermore $f_{i}\left(x, t_{1}, \ldots, t_{k}\right)=0$ for $\left|\left(t_{1}, \ldots, t_{k}\right)\right|$ small then every segregated limiting profile $v_{i}$ is $\mathcal{C}^{0, \alpha}$, for every $\alpha<1$.

Remark 1.8. Collecting together the results of Theorems 1.6 and 1.7. we have that for $s=1 / 2$ the limiting profiles are $\mathcal{C}^{0, \alpha}$, for every $\alpha<1$, when either $a_{i j}=1$ or $p>1$. Since for $s=1 / 2$ we have that $L_{a}=(-\Delta)$, one may then try to apply the arguments contained in [5, Section 2] (see also [1, Section 5]). This should eventually imply that the traces of the limiting profiles are indeed Lipschitz continuous.

\section{Preliminary Results}

We devote this section to some results concerning the operator $L_{a}$ and solutions to some associated differential problem. Most of such results already appeared, even if in slightly different form, in the literature. The interested reader may refer to [9, 26, 27] for further details.

Lemma 2.1 ([9, Lemma 2.7]). If $v$ is a non constant, global solution of $L_{a} v=0$ in $\mathbb{R}^{N+1}$, with the property that

$$
|v(X)| \leq C\left(1+|X|^{\gamma}\right)
$$

then $\gamma \geq \min (2 s, 1)$. If furthermore $v(x,-y)=v(x, y)$ then $\gamma \geq 1$ (and $v$ is $a$ polynomial).

Lemma 2.2 ([27, Proposition 11]). Let $v$ satisfy

$$
\begin{cases}L_{a} v=0 & \text { in } \mathbb{R}_{+}^{N+1} \\ \partial_{\nu}^{a} v=\lambda & \text { on } \mathbb{R}^{N}\end{cases}
$$

for some $\lambda \in \mathbb{R}$, and

$$
|v(X)| \leq C\left(1+|X|^{\gamma}\right),
$$

for some $0 \leq \gamma<\min (2 s, 1)$. Then $v$ is constant.

The two last results we need are based on the following comparison principle.

Lemma 2.3 (Comparison principle). Let $u, v \in H^{1 ; a}\left(B^{+}\right)$satisfy

$$
\left\{\begin{array} { l l } 
{ L _ { a } u \leq 0 } & { \text { in } B _ { 1 } ^ { + } } \\
{ \partial _ { \nu } ^ { a } u \leq - M u ^ { p } + \delta } & { \text { on } \partial ^ { 0 } B _ { 1 } ^ { + } , }
\end{array} \quad \left\{\begin{array}{ll}
L_{a} v \geq 0 & \text { in } B_{1}^{+} \\
\partial_{\nu}^{a} v \geq-M v^{p}+\delta & \text { on } \partial^{0} B_{1}^{+}
\end{array}\right.\right.
$$


respectively. Then $u \leq v$ on $\partial^{+} B_{1}^{+}$implies $u \leq v$ on $B_{1}^{+}$.

Proof. Letting $w=u-v$, we obtain that $w$ is a solution to

$$
\begin{cases}L_{a} w \leq 0 & \text { in } B_{1}^{+} \\ \partial_{\nu}^{a} w \leq-M\left(u^{p}-v^{p}\right) & \text { on } \partial^{0} B_{1}^{+} \\ w \leq 0 & \text { on } \partial^{+} B_{1}^{+} .\end{cases}
$$

Testing the equation with $w^{+}$and recalling that $p>0$ we find

$$
\int_{B_{1}^{+}} y^{a}\left|\nabla w^{+}\right|^{2} \mathrm{~d} x \mathrm{~d} y \leq-M \int_{\partial^{0} B^{+}} \frac{u^{p}-v^{p}}{u-v}\left(w^{+}\right)^{2} \mathrm{~d} x \leq 0 .
$$

Lemma 2.4. Let $M>0$ be any large constant and $\delta>0$ be fixed and let $h \in$ $L^{\infty}\left(\partial^{0} B_{1}^{+}\right)$with $\|h\|_{L^{\infty}} \leq \delta$. Any $v \in H^{1 ; a}\left(B_{1}^{+}\right)$non negative solution to

$$
\begin{cases}L_{a} v \leq 0 & \text { in } B_{1}^{+} \\ \partial_{\nu}^{a} v \leq-M v^{p}+h & \text { on } \partial^{0} B_{1}^{+}\end{cases}
$$

verifies

$$
\sup _{\partial^{0} B_{1 / 2}^{+}} v \leq \frac{1+\delta}{M^{1 / p}} \sup _{\partial^{+} B_{1}^{+}} v .
$$

Sketch of proof. The proof is similar to the one of [27, Lemma 3.2], the only difference being in the choice of the supersolution. For $a \in(-1,1)$ and $p>0$ fixed, let $b=1+(1-a) / p>1$ and $f \in A C(\mathbb{R}) \cap \mathcal{C}^{\infty}(\mathbb{R})$ be defined as

$$
f(x)=c \int_{-\infty}^{x} \frac{1}{\left(1+t^{2}\right)^{b / 2}} \mathrm{~d} t,
$$

where $c$ is chosen in such a way that $f(+\infty)=1$. Then, for some $C>0$, the estimate

$$
(-\Delta)^{s} f(x) \geq-C f(x)^{p}
$$

holds for any $x<0$. For $M>0$, the function $f_{M}(x):=f\left(M^{1 /(2 s)} x\right)$ satisfies

$$
(-\Delta)^{s} f_{M}(x)=M^{2 s /(2 s)}\left[(-\Delta)^{s} f\right]\left(M^{1 /(2 s)} x\right) \geq-C M f_{M}^{p}(x) .
$$

Therefore, if we let

$$
g_{M}(x):=f_{M}(x-1)+f_{M}(-x-1)
$$

then

$$
\left(f_{M}^{p}(x-1)+f_{M}^{p}(-x-1)\right)^{1 / p} \leq c_{p} g_{M},
$$

for some $c_{p}>0$. It follows that, for any $M>0$, it holds

$$
\begin{cases}(-\Delta)^{s} g_{M}(x) \geq-C M g_{M}^{p}(x) & \text { in }(-1,1) \\ g_{M}(x) \geq \frac{1}{2} & \text { in } \mathbb{R} \backslash(-1,1) \\ g_{M}(x) \leq C M^{-1 / p} & \text { in }\left(-\frac{1}{2}, \frac{1}{2}\right) .\end{cases}
$$

The lemma follows by comparison between $v$ and the supersolution (see [10])

$$
w_{\delta}:=\delta \frac{1}{M^{1 / p}}+\int_{\mathbb{R}} y^{1-a} \frac{g_{M}(x-\xi)}{\left(\xi^{2}+y^{2}\right)^{1-a / 2}} \mathrm{~d} \xi .
$$


Lemma 2.5. Let $\lambda>0$ and $v \in H_{\mathrm{loc}}^{1 ; a}\left(\overline{\mathbb{R}_{+}^{N+1}}\right)$ be non negative and satisfy

$$
\begin{cases}L_{a} v=0 & \text { in } \mathbb{R}_{+}^{N+1} \\ \partial_{\nu}^{a} v \leq-\lambda v^{p} & \text { on } \mathbb{R}^{N}\end{cases}
$$

If the Hölder quotient of exponent $\gamma$ of $v$ is uniformly bounded, for some $\gamma \in[0,2 s)$, then $v$ is constant.

Proof. When $p \leq 1$ the lemma follows directly from Lemma 2.4 indeed, by translating and scaling,

$$
v\left(x_{0}, 0\right) \leq \sup _{\partial^{0} B_{r / 2}\left(x_{0}, 0\right)} v \leq \frac{1}{\lambda^{1 / p} r^{2 s / p}} \sup _{\partial^{+} B_{r}\left(x_{0}, 0\right)} v \leq C \frac{1+r^{\gamma}}{r^{2 s / p}} \rightarrow 0 \quad \text { as } r \rightarrow \infty .
$$

When $p>1$, we start by showing that $v$ has a bounded trace on $\mathbb{R}^{N}$. Let us assume, on the contrary, that $v(x, 0)$ is not uniformly bounded from above: by the uniform control on the Hölder seminorm, there exists a sequence $\left\{x_{n}\right\} \subset \mathbb{R}^{N}$ such that

$$
M_{n}:=\inf _{\partial^{0} B_{1}^{+}\left(x_{n}, 0\right)} v^{p-1} \rightarrow+\infty
$$

But then, restricting on $B_{1}^{+}\left(x_{n}, 0\right)$, we have that $v \geq 0$ satisfies

$$
\begin{cases}L_{a} v=0 & \text { in } B_{1}^{+}\left(x_{n}, 0\right) \\ \partial_{\nu}^{a} v \leq-M_{n} v & \text { on } \partial B_{1}^{+}\left(x_{n}, 0\right)\end{cases}
$$

and, thanks to Lemma 2.4 (with exponent 1 instead of $p$ ) and the Hölder continuity, we obtain

$$
\inf _{\partial^{0} B_{1}^{+}\left(x_{n}, 0\right)} v \leq \sup _{\partial^{0} B_{1 / 2}^{+}\left(x_{n}, 0\right)} v \leq \frac{1}{M_{n}} \sup _{\partial^{+} B_{1}^{+}\left(x_{n}, 0\right)} v \leq \frac{1}{M_{n}}\left(\inf _{\partial^{0} B_{1}^{+}\left(x_{n}, 0\right)} v+C\right),
$$

a contradiction. Let now $\left\{x_{n}\right\} \subset \mathbb{R}^{N}$ be a maximizing sequence of $v(x, 0)$, that is

$$
\sup _{x \in \mathbb{R}^{N}} v(x, 0)=\lim _{n \rightarrow \infty} v\left(x_{n}, 0\right)<\infty,
$$

and let us also introduce the sequence of functions

$$
v_{n}(x, y):=v\left(x-x_{n}, y\right)
$$

The functions $v_{n}$ share the same uniform bound in $\mathcal{C}^{0, \gamma}$, so that we can pass to the uniform limit and find a limiting function $\bar{v} \in \mathcal{C}^{0, \gamma}\left(\overline{\mathbb{R}_{+}^{N+1}}\right)$ which satisfies the assumptions of the lemma, its trace on $\mathbb{R}^{N}$ achieving the global maximum at $(0,0)$. Let us denote with $w$ the unique bounded $L_{a}$-harmonic extension of $\bar{v}(x, 0)$ (which is defined since $\bar{v}(x, 0)$ is bounded). We see that the odd extension across $\{y=0\}$ of the difference $w-\bar{v}$ satisfies the assumptions of Lemma 2.1, yielding $\bar{v} \equiv w$. From the equation we deduce that

$$
\partial_{\nu}^{a} \bar{v}(0,0)=-\lambda \bar{v}(0,0)^{p}=-\lambda \sup _{x \in \mathbb{R}^{N}} v(x, 0)^{p} \leq 0
$$

and the Hopf Lemma implies $\bar{v}(0,0)=0$, that is $v \equiv 0$. 


\section{THE BLOW-UP ARGUMENT}

As we mentioned in the introduction, the proof of the a priori uniform $\mathcal{C}^{0, \alpha_{-}}$ bounds of solutions to problem $(P)_{\beta}$ is based on a blow-up argument. To perform this technique, we will assume that the solutions are not a priori bounded in a uniform way in some Hölder norms and then, through a series of lemmas, we will show that this implies the existence of entire solutions to some limiting problem. The scheme of the proof here presented may resemble the one contained for instance in [14] and also 26, 27]. However, in the present situation, some of the steps, which were adopted in the aforementioned papers, actually fail. This phenomenon is consequence of deep differences in the interaction between competition and diffusion features of the models. Once the blow-up procedure is completed, we will reach different contradictions in the next section, depending on the particular choice of $k, p$ and $a_{i j}$ : for the moment, in what follows we will always assume that $p>0$, $a_{i j}>0$ for any $j \neq i$, and that the reaction terms $f_{i, \beta}$ are continuous and map bounded sets into bounded sets, uniformly w.r.t. $\beta>0$ (notice that these are the common assumptions for all the statements in the introduction).

Let $\left\{\mathbf{v}_{\beta}\right\}_{\beta}=\left\{\left(v_{1, \beta}, \ldots, v_{k, \beta}\right)\right\}_{\beta}$ denote a family of positive solutions to problem $(P)_{\beta}$, uniformly bounded in $B_{1}^{+}$. We begin the analysis by recalling the regularity result which holds whenever $\beta$ is finite. For easier notation, we write $B^{+}=B_{1}^{+}$.

Lemma 3.1. For every $0<\alpha<\min (2 s, 1), \bar{m}>0$ and $\bar{\beta}>0$, there exists a constant $C=C(\alpha, \bar{m}, \bar{\beta})$ such that

$$
\left\|\mathbf{v}_{\beta}\right\|_{\mathcal{C}^{0, \alpha}\left(\overline{B_{1 / 2}^{+}}\right)} \leq C,
$$

for every $\mathbf{v}_{\beta}$ solution of problem $(P)_{\beta}$ on $B^{+}$, satisfying

$$
\beta \leq \bar{\beta} \quad \text { and } \quad\left\|\mathbf{v}_{\beta}\right\|_{L^{\infty}\left(B^{+}\right)} \leq \bar{m} .
$$

Sketch of the proof. Since the functions involved are a priori in $L^{\infty}\left(B^{+}\right)$, we can apply the regularity result in [27, Lemma 4.1] to obtain regularity of the solutions in $\mathcal{C}^{0, \alpha}$ spaces for every $\alpha<\min (2 s, 1)$ (see also the proof of [19, Lemma 2.3]).

Let the cut-off function $\eta$ be smooth, with

$$
\eta(X)=\left\{\begin{array}{ll}
1 & X \in B_{1 / 2} \\
0 & X \in \mathbb{R}^{N+1} \backslash B_{1}
\end{array} \quad \text { while } \quad \eta(X) \in(0,1)\right. \text { elsewhere. }
$$

The rest of this section is devoted to the proof of the following proposition.

Proposition 3.2. If there exists $0<\alpha<\min (2 s, 1)$ such that

$$
\sup _{\beta>0}\left|\eta \mathbf{v}_{\beta}\right|_{\mathcal{C}^{0, \alpha}\left(\overline{B^{+}}\right)}=+\infty
$$

then for a suitable choice of $\left\{r_{\beta}\right\}_{\beta} \subset \mathbb{R}^{+}$and $\left\{x_{\beta}^{\prime}\right\}_{\beta} \subset \mathbb{R}^{N}$, the blow-up family

$$
w_{i, \beta}(X):=\eta\left(x_{\beta}^{\prime}, 0\right) \frac{v_{i, \beta}\left(\left(x_{\beta}^{\prime}, 0\right)+r_{\beta} X\right)}{r_{\beta}^{\alpha}\left|\eta \mathbf{v}_{\beta}\right|_{\mathcal{C}^{0, \alpha}}\left(\overline{B^{+}}\right)}
$$

admits a convergent subsequence in the local uniform topology. Moreover the limit $\mathbf{w} \in\left(H_{\text {loc }}^{1 ; a} \cap \mathcal{C}^{0, \alpha}\right)\left(\overline{\mathbb{R}_{+}^{N+1}}\right)$ enjoys the following properties:

(1) each $w_{i}$ is a $L_{a}$-harmonic function of $\mathbb{R}_{+}^{N+1}$; 
(2) at least one component of $\mathbf{w}$ is non constant, and it attains its maximal

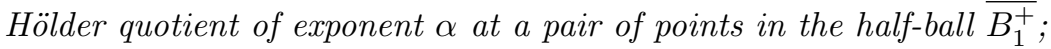

(3) either there exists $M>0$ such that

$$
\partial_{\nu}^{a} w_{i}=-M w_{i}^{p} \sum_{j \neq i} a_{i j} w_{j}^{p} \quad \text { on } \mathbb{R}^{N}
$$

or $\left.w_{i} w_{j}\right|_{y=0}=0$ for every $j \neq i$ and

$$
\partial_{\nu}^{a} w_{i} \leq 0, \quad w_{i} \partial_{\nu}^{a}\left(w_{i}-\sum_{j \neq i} \frac{a_{i j}}{a_{j i}} w_{j}\right)=0 \quad \text { on } \mathbb{R}^{N} .
$$

The proof is divided in several steps. First, we choose any subsequence $\mathbf{v}_{n}:=\mathbf{v}_{\beta_{n}}$ such that

$$
\sup _{n \in \mathbb{N}}\left|\eta \mathbf{v}_{n}\right|_{\mathcal{C}^{0, \alpha}\left(\overline{B^{+}}\right)}=: \sup _{n \in \mathbb{N}} L_{n}=+\infty
$$

where by Lemma 3.1 both $\beta_{n} \rightarrow \infty$ and the Hölder quotients $L_{n}$ are achieved, say

$$
\begin{aligned}
L_{n} & :=\max _{i=1, \ldots, k} \max _{X^{\prime} \neq X^{\prime \prime} \in \overline{B^{+}}} \frac{\left|\left(\eta v_{i, n}\right)\left(X^{\prime}\right)-\left(\eta v_{i, n}\right)\left(X^{\prime \prime}\right)\right|}{\left|X^{\prime}-X^{\prime \prime}\right|^{\alpha}} \\
& =\frac{\left|\left(\eta v_{1, n}\right)\left(X_{n}^{\prime}\right)-\left(\eta v_{1, n}\right)\left(X_{n}^{\prime \prime}\right)\right|}{r_{n}^{\alpha}},
\end{aligned}
$$

where we have written $r_{n}:=\left|X_{n}^{\prime}-X_{n}^{\prime \prime}\right|$. Finally, we are in a position to define the two blow-up sequences we will work with as

$w_{i, n}(X):=\eta\left(x_{n}^{\prime}, 0\right) \frac{v_{i, n}\left(\left(x_{n}^{\prime}, 0\right)+r_{n} X\right)}{L_{n} r_{n}^{\alpha}} \quad$ and $\quad \bar{w}_{i, n}(X):=\frac{\left(\eta v_{i, n}\right)\left(\left(x_{n}^{\prime}, 0\right)+r_{n} X\right)}{L_{n} r_{n}^{\alpha}}$,

both defined on the domain

$$
\tau_{n} B^{+}:=\frac{B^{+}-\left(x_{n}^{\prime}, 0\right)}{r_{n}} .
$$

Accordingly, the corresponding reaction terms can be expressed as

$$
f_{i, n}\left(x, t_{1}, \ldots, t_{k}\right)=r_{n}^{2 s} \frac{\eta\left(x_{n}^{\prime}, 0\right)}{L_{n} r_{n}^{\alpha}} f_{i, \beta_{n}}\left(X_{n}^{\prime}+r_{n} x, t_{1} \frac{L_{n} r_{n}^{\alpha}}{\eta\left(x_{n}^{\prime}, 0\right)}, \ldots, t_{k} \frac{L_{n} r_{n}^{\alpha}}{\eta\left(x_{n}^{\prime}, 0\right)}\right) .
$$

In [26, Section 6] and [27, Section 4] we have analyzed in detail the behavior of the two blow-up sequences in the different case of variational competition. In the following lemma we collect the initial remarks about such sequences, the proof of which is independent of the type of competition. In particular, we have that the domains exhaust the whole $\mathbb{R}_{+}^{N+1}$, and that the two sequences $\left\{\mathbf{w}_{n}\right\}_{n}$ and $\left\{\overline{\mathbf{w}}_{n}\right\}_{n}$ - of which the former satisfies an equation and the latter has uniformly bounded Hölder quotient - are close on any compact.

Lemma 3.3. As $n \rightarrow \infty$ the following assertions hold

(1) $r_{n} \rightarrow 0,\left\|f_{i, n}\right\|_{\infty} \rightarrow 0, \tau_{n} B^{+} \rightarrow \mathbb{R}_{+}^{N+1}$ and $\tau_{n} \partial^{0} B^{+} \rightarrow \mathbb{R}^{N} \times\{0\}$;

(2) the sequence $\left\{\mathbf{w}_{n}\right\}_{n}$ satisfies

$$
\begin{cases}L_{a} w_{i, n}=0 & \text { in } \tau_{n} B^{+} \\ \partial_{\nu}^{a} w_{i, n}=f_{i, n}\left(x, w_{1, n}, \ldots, w_{k, n}\right)-M_{n} w_{i, n}^{p} \sum_{j \neq i} a_{i j} w_{j, n}^{p} & \text { on } \tau_{n} \partial^{0} B^{+},\end{cases}
$$

where

$$
M_{n}=\beta_{n} r_{n}^{2 s}\left(\frac{\eta\left(x_{n}^{\prime}, 0\right)}{L_{n} r_{n}^{\alpha}}\right)^{1-2 p}
$$


(3) the sequence $\left\{\overline{\mathbf{w}}_{n}\right\}_{n}$ has uniformly bounded $\mathcal{C}^{0, \alpha}$-seminorm, the oscillation of the first component in $B_{1}^{+}$being always 1 ;

(4) for any compact $K \subset \mathbb{R}^{N+1}$,

$$
\max _{X \in K \cap \overline{\tau_{n} B^{+}}}\left|\mathbf{w}_{n}(X)-\overline{\mathbf{w}}_{n}(X)\right| \rightarrow 0
$$

(and therefore also $\mathbf{w}_{n}$ has uniformly bounded oscillation on $K$ ).

In the next series of lemmas we are going to show that both sequences converge to the same blow-up limit. To this end, we have to exclude the case in which the sequences are unbounded at the origin: indeed, the uniform boundedness of a sequence at some point is enough, together with points (3) and (4) of the previous lemma, to conclude the convergence (uniform on compact sets) of the two sequences.

Lemma 3.4. For any $r>0$ there exists a constant $C$ such that the estimate

$$
M_{n} \int_{\partial^{0} B_{r}^{+}} \sum_{j \neq i} a_{i j} w_{i, n}^{p+1} w_{j, n}^{p} \mathrm{~d} x \leq C(r)\left(\left|w_{i, n}(0)\right|+1\right)
$$

holds uniformly in $n$.

Proof. Let us consider the quantities

$$
\begin{aligned}
& E(r):=\frac{1}{r^{N+a-1}}\left(\int_{B_{r}^{+}} y^{a}\left|\nabla w_{i, n}\right|^{2}+\int_{\partial^{0} B_{r}^{+}}\left(-f_{i, n} w_{i, n}+M_{n} w_{i, n}^{p+1} \sum_{j \neq i} a_{i j} w_{j, n}^{p}\right)\right) \\
& H(r):=\frac{1}{r^{N+a}} \int_{\partial^{+} B_{r}^{+}} y^{a} w_{i, n}^{2},
\end{aligned}
$$

where $H \in A C(R, 2 R)$, for any $R>0$ fixed and $n$ sufficiently large. If we test equation (3.1) by $w_{i, n}$ itself in the ball $B_{r}^{+}$, we obtain

$$
H^{\prime}(r)=\frac{2}{r^{N+a}} \int_{\partial^{+} B_{r}^{+}} y^{a} w_{i, n} \partial_{\nu} w_{i, n}=\frac{2}{r} E(r),
$$

which can be integrated to infer

$$
H(2 R)-H(R)=\int_{R}^{2 R} \frac{2}{r} E(r) \mathrm{d} r .
$$

On the one hand, the left hand side of of the previous identity can be estimated by recalling that $w_{i, n}$ has uniformly bounded oscillation on any compact set (Lemma 3.3. (4)):

$$
\begin{aligned}
H(2 R)-H(R) & =\int_{\partial^{+} B^{+}} y^{a}\left[w_{i, n}^{2}(2 R X)-w_{i, n}^{2}(R X)\right] \mathrm{d} \sigma \\
& =\left.\int_{\partial^{+} B^{+}} y^{a} w_{i, n}\right|_{R X} ^{2 R X}\left[\left.w_{i, n}\right|_{0} ^{2 R X}+\left.w_{i, n}\right|_{0} ^{R X}+2 w_{i, n}(0)\right] \mathrm{d} \sigma \\
& \leq C(R)\left(\left|w_{i, n}(0)\right|+1\right) .
\end{aligned}
$$


On the other hand, we obtain a lower bound of the right hand side as

$$
\begin{aligned}
\int_{r}^{2 r} \frac{2}{s} E(s) \mathrm{d} s & \geq \min _{s \in[r, 2 r]} E(s) \\
& \geq \frac{1}{r^{N+a-1}}\left(\frac{M_{n}}{2^{N+a}} \int_{\partial^{0} B_{r}^{+}} \sum_{j \neq i} a_{i j} w_{i, n}^{p+1} w_{j, n}^{p} \mathrm{~d} x-\int_{\partial^{0} B_{2 r}^{+}}\left|f_{i, n}\right| w_{i, n} \mathrm{~d} x\right) \\
& \geq C\left(M_{n} \int_{\partial^{0} B_{r}^{+}} \sum_{j \neq i} a_{i j} w_{i, n}^{p+1} w_{j, n}^{p} \mathrm{~d} x-\left\|f_{j, n}\right\|_{L^{\infty}}\left(\left|w_{i, n}(0)\right|+1\right)\right) .
\end{aligned}
$$

Lemma 3.5. If $\bar{w}_{i, n}(0) \rightarrow \infty$ for some $i$, then there exists $C$ such that

$$
M_{n} \bar{w}_{i, n}^{p}(0) \leq C
$$

for a constant $C$ independent of $n$. In particular, $M_{n} \rightarrow 0$.

Proof. Reasoning by contradiction we assume that $M_{n} \bar{w}_{i, n}^{p}(0) \rightarrow \infty$, at least for a subsequence. For any $r>0$ fixed, Lemma 3.3 forces

$$
I_{r, n}:=\inf _{\partial^{0} B_{r}^{+}} M_{n} w_{i, n}^{p} \rightarrow \infty .
$$

From Lemma 3.4 , we directly obtain

$$
M_{n} \inf _{\partial^{0} B_{r}^{+}} w_{i, n}^{p+1} \int_{\partial^{0} B_{r}^{+}} \sum_{j \neq i} a_{i j} w_{j, n}^{p} \mathrm{~d} x \leq C(r)\left(\left|w_{i, n}(0)\right|+1\right)
$$

that is, since $w_{i, n}(0) / w_{i, n}(x) \rightarrow 1$ uniformly in compact sets,

$$
I_{r, n} \int_{\partial^{0} B_{r}^{+}} \sum_{j \neq i} a_{i j} w_{j, n}^{p} \mathrm{~d} x \leq C .
$$

Let $j \neq i$. Since $I_{r, n} \rightarrow \infty$, we deduce that $w_{j, n} \rightarrow 0$ in $L^{p}\left(\partial^{0} B_{r}^{+}\right)$, for every $r$. Therefore Lemma 3.3 implies that both $\left\{\bar{w}_{j, n}\right\}_{n}$ and $\left\{w_{j, n}\right\}_{n}$ converge, uniformly on compact sets, to an $L_{a}$-harmonic function $w_{j, \infty} \in \mathcal{C}^{0, \alpha}\left(\mathbb{R}_{+}^{n+1}\right)$ such that

$$
w_{j, \infty}(x, 0)=0 \quad \text { on } \mathbb{R}^{N} .
$$

The Liouville result in Lemma 2.1 applies to the odd extension of $w_{j, \infty}$ across $\{y=0\}$, yielding

$$
w_{j, \infty} \equiv 0 \quad \text { for } j \neq i .
$$

In particular, by uniform convergence, the unitary Hölder quotient is not achieved by any of the functions $\bar{w}_{j, n}$ for $j \neq i$ and $n$ large enough: it follows that we must have $i=1$.

Now, let us recall that each $w_{j, n}$ with $j \neq 1$ satisfies the inequality

$$
\begin{cases}L_{a} w_{j, n}=0 & \text { in } B_{2 r}^{+} \\ \partial_{\nu}^{a} w_{j, n} \leq\left\|f_{j, n}\right\|_{L^{\infty}\left(B_{2 r}\right)}-a_{j i} I_{2 r, n} w_{j, n}^{p} & \text { in } \partial^{0} B_{2 r}^{+}\end{cases}
$$

so that by Lemma 2.4 we have the estimate

$$
\sup _{\partial^{0} B_{r}^{+}} w_{j, n}^{p} \leq \frac{C(r)}{I_{2 r, n}} \sup _{\partial^{+} B_{2 r}^{+}} w_{j, n}^{p} .
$$


On the other hand, the function $w_{1, n}$ satisfies a boundary condition that can be estimated as

$$
\begin{aligned}
\sup _{\partial^{0} B_{r}^{+}}\left|\partial_{\nu}^{a} w_{1, n}\right| & \leq\left\|f_{1, n}\right\|_{L^{\infty}\left(B_{2 r}\right)}+I_{r, n} \sum_{i \neq 1} a_{i j} \sup _{\partial^{0} B_{r}^{+}} w_{j, n}^{p} \\
& \leq\left\|f_{1, n}\right\|_{L^{\infty}\left(B_{2 r}\right)}+C(r) \frac{I_{r, n}}{I_{2 r, n}} \sum_{i \neq 1} \sup _{\partial^{+} B_{2 r}^{+}} w_{j, n}^{p} \rightarrow 0,
\end{aligned}
$$

where we used the fact that

$$
\inf _{\partial^{0} B_{2 r}^{+}} \bar{w}_{i, n} \leq \inf _{\partial^{0} B_{r}^{+}} \bar{w}_{i, n} \leq \inf _{\partial^{0} B_{2 r}^{+}} \bar{w}_{i, n}+C r^{\alpha} \quad \Longrightarrow \quad \lim _{n \rightarrow \infty} \frac{I_{r, n}}{I_{2 r, n}}=1 .
$$

Let us now introduce the sequences

$$
W_{1, n}(x, y):=w_{1, n}(x, y)-w_{1, n}(0,0), \quad \bar{W}_{1, n}(x, y):=\bar{w}_{1, n}(x, y)-\bar{w}_{1, n}(0,0) .
$$

As before, we can use Lemma 3.3 to prove that both sequences converge to the same $L_{a}$-harmonic function, which is globally Hölder continuous, non constant, and which has trivial conormal derivative on $\mathbb{R}^{N}$, in contradiction with Lemma 2.2 ,

Lemma 3.6. The sequence $\left\{\overline{\mathbf{w}}_{n}(0)\right\}_{n \in \mathbb{N}}$ is bounded.

Proof. By contradiction, let $\left\{\overline{\mathbf{w}}_{n}(0)\right\}_{n \in \mathbb{N}}$ be unbounded. Then, by the previous lemma, $M_{n} \rightarrow 0$. To start with, we claim that for every $j$ there exists a constant $\lambda_{j} \geq 0$ such that, up to subsequences,

$$
M_{n} \bar{w}_{j, n}^{p} \rightarrow \lambda_{j} \quad \text { locally uniformly. }
$$

Indeed, if $\bar{w}_{j, n}(0)$ is bounded this follows by uniform Hölder bounds, with $\lambda_{j}=0$; if it is unbounded, from Lemma 3.5 we obtain that $M_{n} \bar{w}_{j, n}^{p}(0) \rightarrow \lambda_{j}$, while

$$
\sup _{\partial^{0} B_{r}^{+}}\left|M_{n} \bar{w}_{j, n}^{p}-M_{n} \bar{w}_{j, n}^{p}(0)\right|=M_{n} \bar{w}_{j, n}^{p}(0) \sup _{\partial^{0} B_{r}^{+}}\left|\left(\frac{\bar{w}_{j, n}}{\bar{w}_{j, n}(0)}\right)^{p}-1\right| \rightarrow 0 .
$$

Now, let $i$ be such that $w_{i, n}(0)$ is bounded. As usual, we can use Lemma 3.3 to show that $w_{i, n} \rightarrow w_{i, \infty} \in \mathcal{C}^{0, \alpha}\left(\mathbb{R}_{+}^{N+1}\right)$ in the local uniform topology, where, using the claim above, $w_{i, \infty}$ is a solution to

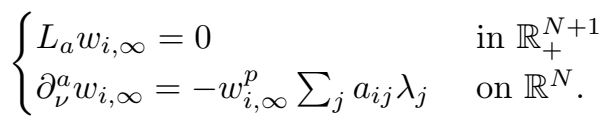

Lemma 2.5 then implies $w_{i, \infty} \equiv 0$ : in particular, we have that $\bar{w}_{1, n}(0)$ is unbounded.

Let us then turn our attention to $w_{1, n}$. Again, if $j$ is such that $\bar{w}_{j, n}(0)$ is bounded, then by the previous discussion $\bar{w}_{j, n} \rightarrow 0$ locally uniformly and

$$
\underbrace{M_{n} \bar{w}_{1, n}^{p}}_{\leq C \text { (Lemma 3.5. }} \bar{w}_{j, n}^{p} \rightarrow 0 .
$$

Otherwise, if $j$ is such that $\bar{w}_{j, n}(0)$ is unbounded, then Lemma 3.4 provides

$$
C \geq M_{n} w_{1, n}(0)^{p} w_{j, n}(0)^{p} \int_{\partial^{0} B_{r}^{+}} \sum_{j \neq 1} a_{1 j} \frac{w_{1, n}^{p+1}}{w_{1, n}(0)^{p}\left(\left|w_{1, n}(0)\right|+1\right)} \frac{w_{j, n}^{p}}{w_{j, n}(0)^{p}} \mathrm{~d} x
$$


so that $M_{n} w_{1, n}(0)^{p} w_{j, n}(0)^{p}$ is uniformly bounded. Since if $\left\{w_{j, n}(0)\right\}_{n \in \mathbb{N}}$ is unbounded then also $\left\{w_{j, n}(x)\right\}_{n \in \mathbb{N}}$ is, for any fixed $x$, and the same argument shows that $M_{n} w_{1, n}(x)^{p} w_{j, n}(x)^{p}$ is bounded. Now,

$$
\begin{aligned}
& M_{n}\left|w_{1, n}(x)^{p} w_{j, n}(x)^{p}-w_{1, n}(0)^{p} w_{j, n}(0)^{p}\right| \\
\leq & M_{n} w_{1, n}(x)^{p} w_{j, n}(x)^{p}\left|1-\frac{w_{j, n}(0)^{p}}{w_{j, n}(x)^{p}}\right|+M_{n} w_{1, n}(0)^{p} w_{j, n}(0)^{p}\left|\frac{w_{1, n}(x)^{p}}{w_{1, n}(0)^{p}}-1\right| \rightarrow 0 .
\end{aligned}
$$

This shows the existence of a constant $\lambda$ such that, at least up to a subsequence,

$$
f_{1, n}-M_{n} \bar{w}_{1, n}^{p} \sum_{j \neq 1} a_{1 j} \bar{w}_{j, n}^{p} \rightarrow \lambda
$$

uniformly on every compact subset of $\mathbb{R}^{N}$, and the same holds true for the sequence $\left\{w_{1, n}\right\}_{n \in \mathbb{N}}$. Thus, as usual, $W_{1, n}=w_{1, n}-w_{1, n}(0)$ converges to $W_{1}$ which is nonconstant, globally Hölder continuous of exponent $\alpha<\min (1,2 s)$, and which solves

$$
\begin{cases}L_{a} W_{1}=0 & \text { in } \mathbb{R}_{+}^{N+1} \\ \partial_{\nu}^{a} W_{1}=\lambda & \text { on } \mathbb{R}^{N}\end{cases}
$$

Invoking Lemma 2.2, we obtain a contradiction.

The boundedness of the sequences $\left\{\overline{\mathbf{w}}_{n}(0)\right\}_{n \in \mathbb{N}}$ implies, by Lemma 3.3 , the convergence of both $\left\{\overline{\mathbf{w}}_{n}\right\}_{n \in \mathbb{N}}$ and $\left\{\mathbf{w}_{n}\right\}_{n \in \mathbb{N}}$ to the same blow-up limit. Reasoning as in the proof of [26, Lemma 6.13], one can show that the convergence is also strong in the natural Sobolev space.

Lemma 3.7. There exists $\mathbf{w} \in\left(H_{\mathrm{loc}}^{1 ; a} \cap \mathcal{C}^{0, \alpha}\right)\left(\overline{\mathbb{R}_{+}^{N+1}}\right)$ such that, up to a subsequence,

$$
\mathbf{w}_{n} \rightarrow \mathbf{w} \text { in }\left(H^{1 ; a} \cap C\right)(K)
$$

for every compact $K \subset \overline{\mathbb{R}_{+}^{N+1}}$. Furthermore, each $w_{i}$ is $L_{a}$-harmonic, and $w_{1}$ is non constant.

Depending on the behavior of the sequence $M_{n}$, the limiting functions w satisfy a different limiting problem: first, we can exclude the case $M_{n} \rightarrow 0$.

Lemma 3.8. There exists $C>0$ such that $M_{n} \geq C$.

Proof. Let us assume that there exists a subsequence $M_{n_{k}}$ that converges to 0 . Passing to the limit in the sequence, we obtain as a limiting problem

$$
\begin{cases}L_{a} w_{i}=0 & \text { in } \mathbb{R}_{+}^{N+1} \\ \partial_{\nu}^{a} w_{i}=0 & \text { on } \mathbb{R}^{N}\end{cases}
$$

By Lemma 2.1 each $w_{i}$ is constant, and this is in contradiction with the fact that $w_{1}$ oscillates in the half-ball $B^{+}$.

Lemma 3.9. If $M_{n} \rightarrow M>0$, then the blow-up profiles $\mathbf{w}$ solve

$$
\begin{cases}L_{a} w_{i}=0 & \text { in } \mathbb{R}_{+}^{N+1} \\ \partial_{\nu}^{a} w_{i}=-M w_{i}^{p} \sum_{j \neq i} a_{i j} w_{j}^{p} & \text { on } \mathbb{R}^{N} .\end{cases}
$$

Proof. This is a direct consequence of Lemma 3.7 .

To conclude the proof of Proposition 3.2 we are left to analyze the case $M_{n} \rightarrow \infty$. 
Lemma 3.10. If $M_{n} \rightarrow \infty$, then the blow-up profiles $\mathbf{w}$ are such that $\left.w_{i} w_{j}\right|_{y=0}=0$, for every $j \neq i$, and

$$
\left\{\begin{array}{l}
\partial_{\nu}^{a} w_{i} \leq 0 \\
\partial_{\nu}^{a}\left(w_{i}-\sum_{j \neq i} \frac{a_{i j}}{a_{j i}} w_{j}\right) \geq 0 \\
w_{i} \partial_{\nu}^{a}\left(w_{i}-\sum_{j \neq i} \frac{a_{i j}}{a_{j i}} w_{j}\right)=0,
\end{array}\right.
$$

where the inequalities are understood in the sense of $\mathbb{R}^{N}$-measures.

Proof. For any nonnegative $\psi \in \mathcal{C}_{0}^{\infty}\left(\mathbb{R}^{N+1}\right)$, we test equation $(3.1)$ to find

$$
0 \leq \int_{\partial^{0} B_{r}} M_{n} w_{i, n}^{p} \sum_{j \neq i} a_{i j} w_{j, n}^{p} \psi \leq \int_{\partial^{0} B_{r}}\left(f_{i, n} \psi-w_{i, n} \partial_{\nu}^{a} \psi\right)-\int_{B_{r}^{+}} w_{i, n} L_{a} \psi .
$$

Since the right hand side is bounded by local uniform convergence, we infer that

$$
M_{n} \int_{K} w_{i, n}^{p} w_{j, n}^{p} \mathrm{~d} x \leq C(K) \quad \forall j \neq i
$$

for any compact set $K \subset \mathbb{R}^{N}$. In particular it follows that, at the limit, $\left.w_{i} w_{j}\right|_{y=0}=$ 0 for every $j \neq i$. Furthermore, the first and the second inequalities in (3.4) follow from equation (3.1) and from the fact that, for every $n$,

$$
\partial_{\nu}^{a}\left(w_{i, n}-\sum_{j \neq i} \frac{a_{i j}}{a_{j i}} w_{j, n}\right)=f_{i, n}-\sum_{j \neq i} \frac{a_{i j}}{a_{j i}} f_{j, n}+M_{n} \sum_{\substack{j \neq i \\ h \neq i, j}} \frac{a_{i j}}{a_{j i}} a_{j h} w_{j, n}^{p} w_{h, n}^{p}
$$

(we recall that the reaction terms $f_{i, n} \rightarrow 0$ uniformly in $\mathbb{R}^{N}$ ). Finally, the identity in (3.4) can be obtained by multiplying the previous equation by $w_{i, n}$, once one can estimate the terms $M_{n} w_{i, n} w_{j, n}^{p} w_{h, n}^{p}$. To this aim, let $\varepsilon>0$, and let us define the (possibly empty) set

$$
\operatorname{supp}_{i}^{\varepsilon}=\left\{x \in \mathbb{R}^{N}: w_{i}(x, 0) \geq \varepsilon\right\} .
$$

We observe that for any $K \subset \mathbb{R}^{N}$ compact set, the local uniform convergence of the sequence $\left\{\mathbf{w}_{n}\right\}$ implies

$$
\begin{cases}w_{i, n}(x, 0) \geq \frac{\varepsilon}{2} & \forall x \in K \cap \operatorname{supp}_{i}^{\varepsilon} \\ w_{i, n}(x, 0) \leq 2 \varepsilon & \forall x \in K \backslash \operatorname{supp}_{i}^{\varepsilon}\end{cases}
$$

for any $n$ large enough. As a consequence

$$
\begin{array}{r}
M_{n} \int_{K} w_{i, n} w_{j, n}^{p} w_{h, n}^{p} \mathrm{~d} x \leq M_{n} \int_{K \backslash \operatorname{supp}_{i}^{\varepsilon}} w_{i, n} w_{j, n}^{p} w_{h, n}^{p} \mathrm{~d} x+M_{n} \int_{K \cap \operatorname{supp}_{i}^{\varepsilon}} w_{i, n} w_{j, n}^{p} w_{h, n}^{p} \mathrm{~d} x \\
\leq M_{n} 2 \varepsilon \int_{K \backslash \operatorname{supp}_{i}^{\varepsilon}} w_{j, n}^{p} w_{h, n}^{p} \mathrm{~d} x+M_{n} \int_{K \cap \operatorname{supp}_{i}^{\varepsilon}} w_{i, n} 2^{2 p} \frac{\left(1+\left\|f_{j, n}\right\|\right)^{p}}{M_{n} \varepsilon^{p}} \frac{\left(1+\left\|f_{h, n}\right\|\right)^{p}}{M_{n} \varepsilon^{p}} \mathrm{~d} x \\
\leq C\left(\varepsilon+\frac{1}{M_{n}} \varepsilon^{-2 p}\right),
\end{array}
$$

where we used estimate 3.5 and Lemma 2.4 to bound the two terms. Choosing $n$ sufficiently large so that $\varepsilon^{-2 p} \leq \varepsilon M_{n}$, we conclude by the arbitrariness of $\varepsilon$ that

$$
\lim _{n \rightarrow \infty} M_{n} \int_{K} w_{i, n} w_{j, n}^{p} w_{h, n}^{p} \mathrm{~d} x=0 \quad \text { for every } i \neq j \neq h .
$$


Corollary 3.11. Let $a_{i j}=1$ for every $i, j$ and $\mathbf{w}$ be a blow-up profile. For every $i \neq j$ the functions $z=w_{i}-w_{j}$ are such that

$$
\begin{cases}L_{a} z^{ \pm} \leq 0 & \text { in } \mathbb{R}_{+}^{N+1} \\ z^{ \pm} \partial_{\nu}^{a} z^{ \pm} \leq 0 & \text { on } \mathbb{R}^{N}\end{cases}
$$

Proof. A subtraction of the equation satisfied by $w_{i, n}$ and $w_{j, n}$ yields

$$
\begin{aligned}
\left(w_{i, n}-w_{j, n}\right)^{ \pm} \partial_{\nu}^{a}\left(w_{i, n}-w_{j, n}\right) & =\left(f_{i, n}-f_{j, n}\right)\left(w_{i, n}-w_{j, n}\right)^{ \pm} \\
& -M_{n} \underbrace{\left(w_{i, n}-w_{j, n}\right)^{ \pm}\left(w_{i, n}^{p}-w_{j, n}^{p}\right)}_{\geq 0} \sum_{h \neq i, j} w_{h, n}^{p} .
\end{aligned}
$$

\section{UNIFORM HÖLDER BOUNDS}

We are ready to show the almost optimal uniform Hölder bounds, in the case of two competing species. This will be a consequence of the following lemma.

Lemma 4.1. Under the assumption of Proposition 3.2, at least three components of the blow-up profile $\mathbf{w}$ are non constant, and all the constant components are trivial.

Proof. We start by observing that each constant component has to be trivial: this is a direct consequence of the segregation condition $\left.w_{i} w_{j}\right|_{y=0}=0$ in the case $M_{n} \rightarrow$ $\infty$, while, if $M_{n} \rightarrow M>0$, it is implied by the boundary condition and the fact that at least $w_{1}$ is non constant.

If $w_{1}$ is the only non constant component, then we obtain a contradiction with Lemma 2.2 since in both cases $M_{n} \rightarrow M$ (Lemma 3.9) and $M_{n} \rightarrow \infty$ (Lemma 3.10), we have $\partial_{\nu}^{a} w_{1}=0$.

Let us now assume that only $w_{1}$ and, say, $w_{2}$ are non constant. Invoking again Lemma 3.9 and 3.10 , we obtain in both cases that

$$
\left\{\begin{array}{lll}
L_{a}\left(a_{21} w_{1}-a_{12} w_{2}\right)=0 & & \text { in } \mathbb{R}_{+}^{N+1} \\
\partial_{\nu}^{a}\left(a_{21} w_{1}-a_{12} w_{2}\right)=0 & & \text { on } \mathbb{R}^{N}
\end{array}\right.
$$

The application of Lemma 2.2 then implies

$$
w_{1}=C+\frac{a_{12}}{a_{21}} w_{2},
$$

where, up to a permutation between $w_{1}$ and $w_{2}$, we may assume that the constant $C$ is non negative. If $M_{n} \rightarrow \infty$, the segregation condition $\left.w_{1} w_{2}\right|_{y=0}=0$ yields

$$
\left.\left(C+\frac{a_{12}}{a_{21}} w_{2}\right) w_{2}\right|_{y=0}=0 \Longrightarrow C=w_{1}=w_{2}=0
$$

a contradiction. In the remaining case, the function $w_{2}$ solves

$$
\begin{cases}L_{a} w_{2}=0 & \text { in } \mathbb{R}_{+}^{N+1} \\ \partial_{\nu}^{a} w_{2}=-M w_{2}^{p}\left(C+\frac{a_{12}}{a_{21}} w_{2}\right)^{p} \leq-C^{\prime} w_{2}^{2 p} & \text { on } \mathbb{R}^{N}\end{cases}
$$

in contradiction with Lemma 2.5 .

Proof of Theorem 1.1. The lemma above, combined with Proposition 3.2 provides all the results in the theorem but the $H^{1 ; a}$ convergence; this last property follows from the uniform Hölder bounds, reasoning as in the proof of Lemma 3.7 
Now we turn to the case of $k \geq 3$ densities. We first prove uniform Hölder bounds with small exponent, when the power $p$ is greater or equal than 1 . In order to quantify such exponent, we need to introduce some notation. For any $\omega \subset \mathbb{S}_{+}^{N}:=\partial^{+} B_{1}^{+}$we consider the first eigenvalue of (the angular part of) $L_{a}$, defined as

$$
\lambda_{1}(\omega)=\inf \left\{\int_{\mathbb{S}_{+}^{N}}|y|^{a}\left|\nabla_{T} u\right|^{2} \mathrm{~d} \sigma: u \equiv 0 \text { on } \mathbb{S}_{+}^{N} \backslash \omega, \int_{\mathbb{S}_{+}^{N}}|y|^{a} u^{2} \mathrm{~d} \sigma=1\right\}
$$

(here $\nabla_{T}$ denotes the tangential part of the gradient), and the associated characteristic function

$$
\gamma(t)=\sqrt{\left(\frac{N-2 s}{2}\right)^{2}+t}-\frac{N-2 s}{2} .
$$

We are ready to state the following Liouville type result.

Proposition 4.2. Under the assumption

$$
p \geq 1,
$$

let $\mathbf{w}$ denote a blow-up limit as in Proposition 3.2, and let

$$
\nu=\nu(s, N):=\inf \left\{\frac{\gamma\left(\lambda_{1}\left(\omega_{1}\right)\right)+\gamma\left(\lambda_{1}\left(\omega_{2}\right)\right)}{2}: \omega_{i} \subset \mathbb{S}_{+}^{N}, \omega_{1} \cap \omega_{2} \cap\{y=0\}=\emptyset\right\} .
$$

If

$$
|\mathbf{w}(X)| \leq C\left(1+|X|^{\alpha}\right), \quad \text { for some } \alpha<\nu,
$$

then $k-1$ components of $\mathbf{w}$ are trivial.

Remark 4.3. As shown in [26, Lemma 2.5], [27, Lemma 2.3], $\nu(s, N)>0$ (and $\nu(s, N) \leq s)$ for every $0<s<1, N \geq 1$.

Proof. The proof is a byproduct of arguments already exploited in 27. The first step consists in obtaining a monotonicity formula of Alt-Caffarelli-Friedman type, with exponent between $\alpha$ and $\nu$. In the case in which $\mathbf{w}$ has segregated traces on $\{y=0\}$, this is [27, Proposition 4]. When $\mathbf{w}$ satisfies a differential system, this can be done as in [27, Proposition 5], with minor changes: namely, by replacing the term $v_{i}^{2} v_{j}^{2}$ with $v_{i}^{p+1} v_{j}^{p}$ (this can be done as far as $p \geq 1$ ).

Once the validity of the monotonicity formula holds, one can deduce a related minimal growth rate for $\mathbf{w}$, which is consistent with the one in the assumption only if all the components but one vanish.

The result above can be improved, also removing the restriction on $p$, in the case of equal competition rates.

Proposition 4.4. Under the assumption

$$
a_{i j}=1 \quad \text { for every } 1 \leq i, j \leq k,
$$

let $\mathbf{w}$ denote a blow-up limit as in Proposition 3.2. and let

$$
\mu=\mu(s, N):=\inf \left\{\frac{\gamma\left(\lambda_{1}\left(\omega_{1}\right)\right)+\gamma\left(\lambda_{1}\left(\omega_{2}\right)\right)}{2}: \omega_{i} \subset \mathbb{S}_{+}^{N}, \omega_{1} \cap \omega_{2}=\emptyset\right\} .
$$

If

$$
|\mathbf{w}(X)| \leq C\left(1+|X|^{\alpha}\right), \quad \text { for some } \alpha<\mu,
$$

then $k-1$ components of $\mathbf{w}$ are trivial. 
Remark 4.5. It is immediate to check that $\mu(s, N) \geq \nu(s, N)$ for every $s, N$. In particular, it is always positive. Were $\mu(s, N)=1$, we would find regularity up to $\alpha_{\text {opt }}$ also in the case $k \geq 3$. As a matter of fact, at the end of this section we will show that $1 / 2 \leq \mu(s, N) \leq 1$ for every $0<s<1, N \geq 1$. Furthermore, it is proved in $[1,8$, that

$$
\mu\left(\frac{1}{2}, N\right)=1, \quad \text { for every } N \geq 1
$$

Proof. We start showing that, for any choice $i \neq j$, if $w_{i}(\cdot, 0) \leq w_{j}(\cdot, 0)$ then $w_{i} \equiv 0$. Indeed, if $\mathbf{w}$ solves the differential system, then

$$
\partial_{\nu}^{a} w_{i} \leq-M w_{i}^{p} w_{j}^{p} \leq-M w_{i}^{2 p},
$$

and the claim follows by Lemma 2.5 in the case of segregated traces, then $w_{i}(\cdot, 0) \equiv$ 0 and one can conclude by applying Lemma 2.1 (to the odd extension of $w_{i}$ across $\{y=0\})$.

On the other hand, let us assume by contradiction that, for some $i \neq j$, the functions $z^{ \pm}:=\left(w_{i}-w_{j}\right)^{ \pm}$are both nontrivial. Then they satisfy the inequalities in Corollary 3.11, and furthermore

$$
\left|z^{ \pm}(X)\right| \leq C\left(1+|X|^{\alpha}\right)
$$

where $\alpha<\mu$. Under these assumptions, we can obtain a contradiction by reasoning as in the proof of Proposition 4.2. To this aim, the only missing ingredient is the following monotonicity formula.

Lemma 4.6. Let $z_{1}, z_{2} \in H^{1 ; a}\left(B_{R}^{+}\left(x_{0}, 0\right)\right)$ be continuous nonnegative functions such that

- $z_{1} z_{2}=0, z_{i}\left(x_{0}, 0\right)=0$;

- for every non negative $\phi \in \mathcal{C}_{0}^{\infty}\left(B_{R}\left(x_{0}, 0\right)\right)$,

$$
\int_{\mathbb{R}_{+}^{N+1}}\left(L_{a} z_{i}\right) z_{i} \phi \mathrm{d} x \mathrm{~d} y+\int_{\mathbb{R}^{N}}\left(\partial_{\nu}^{a} z_{i}\right) z_{i} \phi \mathrm{d} x=\int_{\mathbb{R}_{+}^{N+1}} y^{a} \nabla z_{i} \cdot \nabla\left(z_{i} \phi\right) \mathrm{d} x \mathrm{~d} y \leq 0 .
$$

Then

$$
\Phi(r):=\prod_{i=1}^{2} \frac{1}{r^{2 \mu}} \int_{B_{r}^{+}\left(x_{0}, 0\right)} \frac{y^{a}\left|\nabla z_{i}\right|^{2}}{\left|X-\left(x_{0}, 0\right)\right|^{N-2 s}} \mathrm{~d} x \mathrm{~d} y
$$

is monotone non decreasing in $r$ for $r \in(0, R)$, where $\mu$ is defined as in Proposition 4.4.

Proof. First of all we observe that, up to an even extension of the functions $z_{i}$ across $\{y=0\}$, the formula above is implied by the analogous one stated on the whole $B_{r}$. This latter formula, when $s=1 / 2$, is nothing but the classical Alt-CaffarelliFriedman one [1. On the other hand, when $s \neq 1 / 2$, its proof resemble the usual one, as done for instance in [8] (see also [27, Section 2] for further details).

To conclude the proof of Theorem 1.6, we provide the following rough elementary estimate of $\mu(s, N)$ for $s \neq 1 / 2$.

Lemma 4.7. For every $0<s<1$ and $N \geq 1$ it holds

$$
\mu(s, N) \geq \frac{1}{2} \text {. }
$$


Proof. By trivial extension to higher dimensions of the eigenfunctions involved, it is easy to prove that $\mu$ is decreasing with respect to $N$, thus we can assume $N \geq 2$.

Let $\omega_{1}, \omega_{2} \subset \mathbb{S}_{+}^{N}, \omega_{1} \cap \omega_{2}=\emptyset$, and let $\phi_{i} \in H^{1 ; a}\left(\mathbb{S}_{+}^{N}\right)$ be the first eigenfunction associated to $\lambda_{1}\left(\omega_{i}\right)$ enjoying the normalization

$$
\int_{\mathbb{S}^{N}}|y|^{a} \phi_{i}^{2} \mathrm{~d} \sigma=1 \quad \text { for } i=1,2 .
$$

If $\mathcal{R}$ denotes the Rayleigh quotient associated to $\lambda_{1}$, then we have that

$$
\lambda_{2}\left(\mathbb{S}_{+}^{N}\right):=\inf _{\substack{V \subset H^{1 ; a}\left(\mathbb{S}_{+}^{N}\right) \\ \operatorname{dim} V \geq 2}} \max _{V} \mathcal{R} \leq \max _{\vartheta} \mathcal{R}\left(\phi_{1} \cos \vartheta+\phi_{2} \sin \vartheta\right) \leq \max \left(\lambda_{1}\left(\omega_{1}\right), \lambda_{1}\left(\omega_{2}\right)\right) .
$$

By monotonicity of $\gamma$ we obtain that

$$
\mu(s, N)=\inf _{\omega_{1} \cap \omega_{2}=\emptyset} \frac{\gamma\left(\lambda_{1}\left(\omega_{1}\right)\right)+\gamma\left(\lambda_{1}\left(\omega_{2}\right)\right)}{2} \geq \frac{1}{2} \gamma\left(\lambda_{2}\left(\mathbb{S}_{+}^{N}\right)\right) .
$$

To conclude the proof, we show that $\gamma\left(\lambda_{2}\left(\mathbb{S}_{+}^{N}\right)\right)=1$. Indeed, let $\psi_{2}$ be a second eigenfunction. Then its conormal derivative on $\partial \mathbb{S}_{+}^{N}$ is identically zero, and it can be extended in an even way across $\{y=0\}$ to an eigenfunction of $\mathbb{S}^{N}$. Moreover, by the well known properties of $\gamma$, we have that the function

$$
v(X)=|X|^{\gamma\left(\lambda_{2}\left(\mathbb{S}_{+}^{N}\right)\right)} \psi_{2}\left(\frac{X}{|X|}\right)
$$

is $L_{a}$-harmonic up to 0 (this is true, actually, because we are assuming $N \geq 2$ ), is $y$-even, and has bounded growth. By Lemma 2.1 we deduce that, up to a rotation in the $x$ plane, $v=x_{1}$, concluding the proof.

Proof of Theorem 1.6. The uniform Hölder bounds with exponent $\alpha^{*}$ are obtained by combining Lemma 4.1 with either Proposition $4.2\left(\right.$ with $\left.\alpha^{*}=\min (2 s, \nu(s, N))\right)$ or Proposition 4.4 (with $\alpha^{*}=\min (2 s, \mu(s, N))$ ), respectively. In the second case, the exact value of $\alpha^{*}$ is provided by Remark 4.5 when $s=1 / 2$, and by Lemma 4.7 when $s<1 / 4$.

Remark 4.8. By comparison with the nodal partition of $\mathbb{S}_{+}^{N}$ associated to the homogeneous, $L_{a}$-harmonic function $v(x, y)=x_{1}$, we infer that

$$
\mu(s, N) \leq 1 \text {. }
$$

\section{Further PROPERTIES OF THE SEGREGATION PROFILES}

In this last section we deal with the proof of Theorems 1.2 and 1.7 . Together with the previous assumptions, in what follows we further suppose that the reaction terms $f_{i, \beta} \rightarrow f_{i}$ as $\beta \rightarrow \infty$, uniformly on compact sets, with $f_{i}$ Lipschitz continuous.

As a result of the previous sections, we have shown that $L^{\infty}$ uniform bounds on a family of solutions to the problem $(P)_{\beta}$ is enough to ensure equicontinuity of the family independently from the competition parameter $\beta$. Reasoning as in the proof of Lemmas 3.7 and 3.10 we deduce the following result.

Proposition 5.1. Any sequence $\left\{\mathbf{v}_{\beta_{n}}\right\}_{n \in \mathbb{N}}, \beta_{n} \rightarrow \infty$, of solutions to $(P)_{\beta}$ which is uniformly bounded in $L^{\infty}\left(B^{+}\right)$admits a subsequence which converges to a limiting 
profile $\mathbf{v} \in\left(H^{1 ; a} \cap \mathcal{C}^{0, \alpha}\right)_{\text {loc }}\left(B^{+}\right)$, for some $\alpha>0$. Moreover

$$
\begin{cases}L_{a} v_{i}=0 & \text { in } B^{+}, \\ \partial_{\nu}^{a} v_{i} \leq f_{i}\left(x, v_{1}, \ldots, v_{k}\right) & \\ \partial_{\nu}^{a}\left(v_{i}-\sum_{j \neq i} \frac{a_{i j}}{a_{j i}} v_{j}\right) \geq f_{i}-\sum_{j \neq i} \frac{a_{i j}}{a_{j i}} f_{j} & \text { on } \partial^{0} B^{+}, \\ v_{i} \cdot\left[\partial_{\nu}^{a}\left(v_{i}-\sum_{j \neq i} \frac{a_{i j}}{a_{j i}} v_{j}\right)-f_{i}+\sum_{j \neq i} \frac{a_{i j}}{a_{j i}} f_{j}\right]=0 & \end{cases}
$$

and $v_{i}(x, 0) \cdot v_{j}(x, 0) \equiv 0$ for every $j \neq i$.

After Proposition 5.1. the optimal regularity for the case of two densities is almost straightforward.

Proof of Theorem 1.2. For a limiting profile $\mathbf{v}=\left(v_{1}, v_{2}\right)$, let $w=a_{21} v_{1}-a_{12} v_{2}$. Then Proposition 5.1 implies that

$$
v_{1}(x, 0)=\frac{1}{a_{21}} w^{+}, \quad v_{2}(x, 0)=\frac{1}{a_{12}} w^{-},
$$

and

$$
\begin{cases}L_{a} w=0 & \text { in } B^{+}, \\ \partial_{\nu}^{a} w=g(w) & \text { on } \partial^{0} B^{+},\end{cases}
$$

where

$$
g(x, t):=a_{21} f_{1}\left(x, \frac{1}{a_{21}} t^{+}, \frac{1}{a_{12}} t^{-}\right)-a_{12} f_{2}\left(x, \frac{1}{a_{21}} t^{+}, \frac{1}{a_{12}} t^{-}\right)
$$

is Lipschitz continuous. As a consequence, standard regularity (e.g. 24, Proposition 2.8], 19. Lemma 2.1]) applies, providing that $w \in \mathcal{C}^{1, \alpha}$ and thus $u_{1}, u_{2}$ are Lipschitz continuous.

Remark 5.2. An important consequence of the argument above is that whenever there are only two species that are segregated, under suitable growth conditions about $f_{1}, f_{2}$ the corresponding free boundary

$$
\Gamma:=\left\{x \in \partial^{0} B: v_{1}(x, 0)=v_{2}(x, 0)=0\right\}
$$

is a closed set of empty interior (in the $N$ dimensional topology). Indeed $w=$ $a_{21} v_{1}-a_{12} v_{2}$ satisfies a semilinear equation for which unique continuation holds, see [20, Theorems 1.4, 4.1].

We are left to deal with the case $k \geq 3$ for the half-laplacian, i.e.

$$
s=\frac{1}{2} \text {. }
$$

In this case, by Theorem 1.6 we already know that, when $a_{i j}=1$, the traces of the limiting profiles enjoy almost Lipschitz continuity on $K \cap\{y \geq 0\}$, for every compact $K \subset B$. We are going to show that the same holds also for general $a_{i j}$, when there are no internal reaction terms in a neighborhood of the free boundary. More precisely, we assume that the Lipschitz continuous functions $f_{i}$ are such that

$$
f_{i}\left(x, t_{1}, \ldots, t_{k}\right) \equiv 0 \quad \text { whenever }\left|\left(t_{1}, \ldots, t_{k}\right)\right|<\theta,
$$

for some $\theta>0$ (such assumption can be weakened, but we prefer to avoid further technicalities at this point). Finally, $K \subset B$ will denote a fixed compact set. 
Remark 5.3. As before, since the components of a limiting profile $\mathbf{v}$ are harmonic on $B^{+}$, we have that its regularity on $K$ is directly connected to the regularity of the same function in $K \cap\{0 \leq y<\varepsilon\}$ for arbitrarily small $\varepsilon>0$.

Definition 5.4. For any function $\mathbf{v} \in H^{1} \cap \mathcal{C}\left(B^{+} ; \mathbb{R}^{k}\right)$ which satisfies (5.1) (with $s=1 / 2)$, we let $\hat{\mathbf{v}}:=\left(\hat{v}_{1}, \ldots, \hat{v}_{k}\right)$ where

$$
\hat{v}_{i}(x, y)=v_{i}(x, y)-\sum_{j \neq i} \frac{a_{i j}}{a_{j i}} v_{j}(x, y) .
$$

To clarify the effect of the segregation condition, we introduce the definition of multiplicity of boundary points.

Definition 5.5. We define the multiplicity of a point $x \in \partial^{0} B^{+}$as

$$
m(x):=\sharp\left\{i: \mathcal{H}^{N}\left(\left\{v_{i}(x, 0)>0\right\} \cap \partial^{0} B_{r}(x, 0)\right)>0 . \forall r>0\right\} .
$$

We start with a result about the regularity of low multiplicity points.

Lemma 5.6. If $K \cap\{y=0\} \subset\{x: m(x) \leq 1\}$ then $\mathbf{v} \in \mathcal{C}^{1,1 / 2}(K \cap\{y \geq 0\})$.

Proof. According to Remark 5.3 , we will show local regularity of the functions in $B_{r}^{+}\left(x_{0}, 0\right)$, where $r$ is small and $m\left(x_{0}\right) \leq 1$. We have three possibilities.

Case 1: $m\left(x_{0}\right)=0$. in this case, $\left.\mathbf{v}\right|_{\partial^{0} B_{r}\left(x_{0}, 0\right)} \equiv 0$, and the result is standard.

Case 2: $m\left(x_{0}\right)=1$ and $v_{i}\left(x_{0}, 0\right)>0$. By continuity of $v_{i}$, we can assume that $\left.v_{i}\right|_{\partial^{0} B_{r}\left(x_{0}, 0\right)}>0$, while by the segregation condition $\left.v_{j}\right|_{\partial^{0} B_{r}\left(x_{0}, 0\right)} \equiv 0$ for every $j \neq i$. Let $\hat{\mathbf{v}}$ be as in Definition 5.4. Since in this case $\hat{v}_{i}=v_{i}$ on $\partial^{0} B_{r}\left(x_{0}, 0\right)$, it follows from (5.1) that

$$
\begin{cases}-\Delta \hat{v}_{i}=0 & \text { in } B_{r}^{+}\left(x_{0}, 0\right) \\ \partial_{\nu} \hat{v}_{i}=f\left(x, 0, \ldots, \hat{v}_{i}, \ldots, 0\right) & \text { in } \partial^{0} B_{r}^{+}\left(x_{0}, 0\right) .\end{cases}
$$

The regularity of $\hat{v}_{i}$ (and thus of $v_{i}$ ) follows by the well established regularity theory of the semilinear Steklov problem.

Case 3: $m\left(x_{0}\right)=1$ and the non trivial function $v_{i}$ is such that $v_{i}\left(x_{0}, 0\right)=0$. Also in this case we can assume $\left.v_{j}\right|_{\partial^{0} B_{r}\left(x_{0}, 0\right)} \equiv 0$ for $j \neq i$ and, as before, $v_{i}=\hat{v}_{i} \geq 0$ on $\partial^{0} B_{r}\left(x_{0}, 0\right)$. By continuity of $v_{i}$, we can also assume that $f_{i}=0$ in $\partial^{0} B_{r}\left(x_{0}, 0\right)$. It follows that

$$
\begin{cases}-\Delta \hat{v}_{i}=0 & \text { in } B_{r}\left(x_{0}, 0\right) \\ \partial_{\nu} \hat{v}_{i}=0 & \text { in } \partial^{0} B_{r}\left(x_{0}, 0\right) \cap\left\{\left.\hat{v}_{i}\right|_{y=0}>0\right\} \\ \partial_{\nu} \hat{v}_{i} \geq 0 & \text { in } \partial^{0} B_{r}\left(x_{0}, 0\right) .\end{cases}
$$

As a consequence, $\hat{v}_{i}$ is a solution to the zero thin obstacle problem, for which $\mathcal{C}^{1,1 / 2}$ regularity (up to the obstacle) has been obtained in [2, Theorem 5].

Remark 5.7. The analogous of the previous lemma holds true also when $s \neq 1 / 2$, in which case $\mathcal{C}^{1, s}$ regularity can be shown, as a consequence of 9 .

Now, for $X \in B$, we introduce the the Morrey quotient associated to $\mathbf{v}$ as

$$
\Phi(X, r):=\frac{1}{r^{N+1-2 \varepsilon}} \int_{B_{r}(X) \cap B^{+}} \sum_{i=1}^{k}\left|\nabla v_{i}\right|^{2} \mathrm{~d} x \mathrm{~d} y .
$$

It is well known that if $\Phi$ is uniformly bounded for any $X \in K \cap\{y \geq 0\}$ and $r<\operatorname{dist}\left(K, \partial^{+} B^{+}\right)$, then $\mathbf{v}$ is Hölder continuous of exponent $1-\varepsilon$ in $K \cap\{y \geq 0\}$. 
Thus the proof of Theorem 1.7 is based on the contradictory assumption that, for some $\varepsilon>0$, there is a sequence $\left\{\left(X_{n}, r_{n}\right)\right\}_{n}$ such that $X_{n} \in K, r_{n}>0$ and

$$
\lim _{n \rightarrow \infty} \Phi\left(X_{n}, r_{n}\right)=\infty
$$

To reach a contradiction we will use the following technical lemma.

Lemma 5.8 ([15, Lemma 8.2]). Let $\Omega \subset \mathbb{R}^{N+1}$ and $v \in H^{1}(\Omega)$ and let

$$
\Phi(X, r):=\frac{1}{r^{N+1-2 \varepsilon}} \int_{B_{r}(X) \cap \Omega}|\nabla v|^{2} \mathrm{~d} x \mathrm{~d} y .
$$

If $\left(X_{n}, r_{n}\right) \subset \bar{\Omega} \times \mathbb{R}^{+}$is a sequence such that $\Phi\left(X_{n}, r_{n}\right) \rightarrow \infty$, then $r_{n} \rightarrow 0$ and

(1) there exists $\left\{r_{n}^{\prime}\right\} \subset \mathbb{R}^{+}$such that $\phi\left(X_{n}, r_{n}^{\prime}\right) \rightarrow \infty$ and

$$
\int_{\partial B_{r_{n}^{\prime}}\left(X_{n}\right) \cap \Omega}|\nabla v|^{2} \leq \frac{N+1-2 \varepsilon}{r_{n}^{\prime}} \int_{B_{r_{n}^{\prime}}\left(X_{n}\right) \cap \Omega}|\nabla v|^{2}
$$

(2) if $A \subset \bar{\Omega}$ and

$$
\operatorname{dist}\left(X_{n}, A\right) \leq C r_{n}
$$

then there exists a sequence $\left\{\left(X_{n}^{\prime}, r_{n}^{\prime}\right)\right\}$ such that $\phi\left(X_{n}^{\prime}, r_{n}^{\prime}\right) \rightarrow \infty$ and $X_{n}^{\prime} \in$ A for every $n$.

Proof of Theorem 1.7. Using the second point of Lemma 5.8, together with Remark 5.3 . we can assume without loss of generality that the contradictory assumption (5.2) holds for $\partial^{0} B^{+} \ni X_{n}=:\left(x_{n}, 0\right)$, for every $n$. Lemma 5.8 also implies that $r_{n} \rightarrow 0$, and that we can assume estimate (5.3) to hold for any $n$, with $\Omega=\{y>0\}$. Furthermore, since $\mathbf{v} \in H^{1}\left(B^{+}\right)$, the function $r \mapsto \Phi\left(\left(x_{n}, 0\right), r\right)$ is continuous for $r>0$ and it is uniformly bounded for $r$ faraway from 0 : as a consequence we can assume that

$$
\Phi\left(\left(x_{n}, 0\right), r\right) \leq C \Phi\left(\left(x_{n}, 0\right), r_{n}\right) \quad \forall r_{n}<r<\operatorname{dist}\left(K, \partial^{+} B^{+}\right),
$$

for some constat $C$. Finally, by Lemmas 5.6 and 5.8 we can assume $m\left(x_{n}\right) \geq 2$ for $n$ large, and thus $f_{i}\left(\cdot, v_{i}\right) \equiv 0$ on $B_{r_{n}}^{+}\left(\left(x_{n}, 0\right)\right)$.

Let us introduce a sequence of scaled function $\mathbf{v}_{n}$ defined as

$$
v_{i, n}(X):=\frac{1}{\Phi\left(\left(x_{n}, 0\right), r_{n}\right)^{1 / 2} r_{n}^{1-\varepsilon}} v_{i}\left(\left(x_{n}, 0\right)+r_{n} X\right) \quad \text { for } X \in B .
$$

By assumptions, $\left\|\nabla \mathbf{v}_{n}\right\|_{L^{2}\left(B^{+}\right)}=1$ for every $n$, and

$$
\frac{1}{r^{N+1-2 \varepsilon}} \int_{B_{r}^{+}} \sum_{i=1}^{k}\left|\nabla v_{i, n}\right|^{2} \leq C \quad \forall 1<r<r_{n}^{-1} \operatorname{dist}\left(K, \partial^{+} B^{+}\right) .
$$

We divide the rest of the proof in a number of steps.

Step 1: also $\left\|\mathbf{v}_{n}\right\|_{L^{2}\left(B^{+}\right)}$is uniformly bounded. We argue by contradiction, assuming that $\left\|\mathbf{v}_{n}\right\|_{L^{2}\left(B^{+}\right)} \rightarrow \infty$. Letting

$$
\mathbf{u}_{n}:=\left\|\mathbf{v}_{n}\right\|_{L^{2}\left(B^{+}\right)}^{-1} \mathbf{v}_{n}
$$

we have that $\left\|\mathbf{u}_{n}\right\|_{L^{2}\left(B^{+}\right)}=1$, while $\left\|\nabla \mathbf{u}_{n}\right\|_{L^{2}\left(B^{+}\right)} \rightarrow 0$ : there exists $\mathbf{d} \in \mathbb{R}^{k}$ such that

$$
\mathbf{u}_{n} \rightarrow \mathbf{d} \quad \text { in } H^{1}\left(B^{+}\right)
$$


Using the segregation condition $\left.v_{i, n} \cdot v_{j, n}\right|_{y=0}=0$, which passes to the strong limit, we infer that only one among the constant $d_{i}$ may be non trivial, say $d_{1}>0$. But recalling that the even extension of $\hat{v}_{i, n}$ across $\{y=0\}$ is superharmonic, we find

$$
\hat{v}_{1, n}(0)=0 \Longrightarrow \int_{B^{+}} \sum_{j \neq 1} \frac{a_{i j}}{a_{j i}} v_{j, n} \geq \int_{B^{+}} v_{1, n}
$$

a contradiction, passing to the strong limit in $H^{1}\left(B^{+}\right)$.

Step 2: the sequence $\mathbf{v}_{n}$ admits a nontrivial weak limit $\overline{\mathbf{v}} \in H_{\mathrm{loc}}^{1}\left(\overline{\mathbb{R}_{+}^{N+1}}\right)$. From Step 1 and the uniform estimate (5.4) we infer the weak convergence; let us show that $\overline{\mathbf{v}}$ is non trivial. To this end, we recall that

$$
\begin{cases}-\Delta v_{i, n}=0 & \text { in } B^{+} \\ v_{i, n} \partial_{\nu} v_{i, n} \leq 0 & \text { on } \partial^{0} B^{+}\end{cases}
$$

Testing the equation against $v_{i, n}$ and summing over $i$, we have

$$
\int_{B^{+}}\left|\nabla \mathbf{v}_{n}\right|^{2} \leq \int_{\partial^{+} B^{+}} \sum_{i=1}^{k} v_{i, n} \partial_{\nu} v_{i, n} \leq\left(\int_{\partial^{+} B^{+}}\left|\mathbf{v}_{n}\right|^{2} \cdot \int_{\partial^{+} B^{+}}\left|\nabla \mathbf{v}_{n}\right|^{2}\right)^{1 / 2} .
$$

Were $\overline{\mathbf{v}}$ trivial, the right hand side would go to zero thanks to the compact embedding of the trace operator and the uniform estimate $(5.3)$, which is scaling invariant. This would imply strong convergence, in contradiction with the fact that the $L^{2}$ norm of $\nabla \mathbf{v}_{n}$ is equal to 1.

Step 3: $\overline{\mathbf{v}}(x, 0) \equiv 0$ on $\mathbb{R}^{N}$. Let us consider the sequence $\hat{\mathbf{v}}_{n}$ (recall Definition 5.4). From (5.1) (in the case $s=1 / 2)$, we know that the pair $\left(\hat{v}_{i}^{+}, \hat{v}_{i}^{-}\right)$is made of two continuous, subharmonic, nonnegative functions such that $\hat{v}_{i}^{+} \cdot \hat{v}_{i}^{-}=0$ in $\mathbb{R}^{N+1}$, with nonpositive normal derivative on $\mathbb{R}^{N}$. As a result, they satisfy the assumption of the Alt-Caffarelli-Friedmann monotonicity formula (Lemma 4.6 with $a=0$ and $\mu=1$ ), from which we obtain

$$
\begin{aligned}
& \frac{1}{r^{N+1}} \int_{B_{r}^{+}\left(x_{n}, 0\right)}\left|\nabla \hat{v}_{i}^{+}\right|^{2} \mathrm{~d} x \mathrm{~d} y \cdot \frac{1}{r^{N+1}} \int_{B_{r_{n}}^{+}\left(x_{n}, 0\right)}\left|\nabla \hat{v}_{i}^{-}\right|^{2} \mathrm{~d} x \mathrm{~d} y \leq \\
& \frac{1}{r^{2}} \int_{B_{r}^{+}\left(x_{n}, 0\right)} \frac{\left|\nabla \hat{v}_{i}^{+}\right|^{2}}{\left|X-\left(x_{n}, 0\right)\right|^{N-1}} \mathrm{~d} x \mathrm{~d} y \cdot \frac{1}{r^{2}} \int_{B_{r}^{+}\left(x_{n}, 0\right)} \frac{\left|\nabla \hat{v}_{i}^{-}\right|^{2}}{\left|X-\left(x_{n}, 0\right)\right|^{N-1}} \mathrm{~d} x \mathrm{~d} y \leq C,
\end{aligned}
$$

that is

$$
\frac{1}{r^{N+1-2 \varepsilon}} \int_{B_{r}^{+}\left(x_{n}, 0\right)}\left|\nabla \hat{v}_{i}^{+}\right|^{2} \mathrm{~d} x \mathrm{~d} y \cdot \frac{1}{r^{N+1-2 \varepsilon}} \int_{B_{r}^{+}\left(x_{n}, 0\right)}\left|\nabla \hat{v}_{i}^{-}\right|^{2} \leq C r^{4 \varepsilon} .
$$

Hence at most one of the two Morrey quotients can be unbounded. Moreover, by the triangular inequality, the possibly unbounded one diverges at most at the same rate of $\Phi\left(\left(x_{n}, 0\right), r_{n}\right)$. Scaling to $\left(\hat{v}_{i, n}^{+}, \hat{v}_{i, n}^{-}\right)$we can distinguish among three different cases:

- both $\left\|\nabla \hat{v}_{i, n}^{+}\right\|_{L^{2}\left(B_{r}\right)}$ and $\left\|\nabla \hat{v}_{i, n}^{-}\right\|_{L^{2}\left(B_{r}\right)}$ are infinitesimal. In this situation, we have that there exists $c \geq 0$ such that $\hat{v}_{i, n} \rightarrow c$. Since by even extension

$$
\left\{\begin{array}{l}
-\Delta \hat{v}_{i, n} \geq 0 \quad \text { in } B_{r} \\
\hat{v}_{i, n}(0,0)=0
\end{array} \quad \Longrightarrow \int_{B_{r}} \hat{v}_{i, n} \leq 0\right.
$$

we have that $\hat{v}_{i, n} \rightarrow c \leq 0$; 
- there exists $c>0$ such that $\left\|\nabla \hat{v}_{i, n}^{+}\right\|_{L^{2}\left(B_{r}\right)} \geq c>0$ while $\left\|\nabla \hat{v}_{i, n}^{-}\right\|_{L^{2}\left(B_{r}\right)} \rightarrow 0$. Testing the equation

$$
\begin{cases}-\Delta \hat{v}_{i, n}^{+} \leq 0 & \text { in } B_{r}^{+} \\ \hat{v}_{i, n}^{+} \partial_{\nu} \hat{v}_{i, n}^{+} \leq 0 & \text { on } \partial^{0} B_{r}^{+}\end{cases}
$$

with $\hat{v}_{i, n}^{+}$we obtain that in the limit $\hat{v}_{i, n}^{+} \rightarrow \hat{v}_{i} \neq 0$, and thus $\hat{v}_{i, n}^{-} \rightarrow 0$ strongly in $H^{1}\left(B_{r}\right)$. Using again the superharmonicity of $\hat{v}_{i, n}$ as before, we conclude that $\hat{v}_{i, n} \rightarrow 0$, in contradiction with $\hat{v}_{i} \neq 0$

- there exists $c>0$ such that $\left\|\nabla \hat{v}_{i, n}^{-}\right\|_{L^{2}\left(B_{r}\right)} \geq c>0$ while $\left\|\nabla \hat{v}_{i, n}^{+}\right\|_{L^{2}\left(B_{r}\right)} \rightarrow 0$. Reasoning as in the previous case, we obtain that $\hat{v}_{i, n}^{+} \rightarrow 0$ strongly in $H^{1}\left(B_{r}\right)$, thus $\hat{v}_{i, n} \rightarrow \hat{v}_{i} \leq 0$.

In any case, $\hat{v}_{i, n}^{+} \rightarrow 0$ and $\hat{v}_{i, n} \rightarrow \hat{v}_{i} \leq 0$ in $H_{\text {loc }}^{1}\left(\mathbb{R}_{+}^{N+1}\right)$ for all $i$, implying in particular that $\left.\left.v_{i, n}\right|_{y=0} \rightarrow \bar{v}_{i}\right|_{y=0}=0$ in $H_{\text {loc }}^{1 / 2}\left(\mathbb{R}^{N}\right)$.

Conclusion. If we extend $\overline{\mathbf{v}}$ evenly across $\{y=0\}$, we obtain a $k$-tuple of harmonic functions defined on $\mathbb{R}^{N+1}$ for which $\Phi(0, r) \leq C$ for all $r \geq 1$. From the Morrey inequality, we have that for any $X \in \mathbb{R}^{N+1},|X| \geq 1$,

$$
|\overline{\mathbf{v}}(X)-\overline{\mathbf{v}}(0)| \leq C|X|^{1-\frac{N+1}{2}}\|\nabla \overline{\mathbf{v}}\|_{L^{2}\left(B_{2|X|}\right)} .
$$

As a result, we have

$$
|\overline{\mathbf{v}}(X)-\overline{\mathbf{v}}(0)| \leq C|X|^{1-\varepsilon}
$$

for every $|X| \geq 1$, in contradiction with the fact that $\mathbf{v}$ is harmonic in $\mathbb{R}^{N+1}$ and non trivial, thanks to the classical Liouville theorem.

Remark 5.9. More general nonlinearities should be addressable, using similar arguments as before, once a generalization of the Caffarelli-Jerison-Kenig almost monotonicity formula [4] to this setting were available.

Remark 5.10. The case $s \neq 1 / 2$ could follow as a generalization of the previous proof, if not for the fact that, at the moment, no exact Alt-Caffarelli-Friedman monotonicity formula is available, in this setting: one could only show, by Lemma 4.6 and 4.7 , the $\mathcal{C}^{0, \alpha}$ continuity of the limiting profiles, for every $\alpha<2 s$ and $\alpha \leq \mu$.

\section{REFERENCES}

[1] H. W. Alt, L. A. Caffarelli, and A. Friedman. Variational problems with two phases and their free boundaries. Trans. Amer. Math. Soc., 282(2):431-461, 1984.

[2] I. Athanasopoulos and L. A. Caffarelli. Optimal regularity of lower-dimensional obstacle problems. Journal of Mathematical Sciences, 132(3):274-284, 2006.

[3] M. Bonforte and J. L. Vázquez. Quantitative local and global a priori estimates for fractional nonlinear diffusion equations. Adv. Math., 250:242-284, 2014.

[4] L. A. Caffarelli, D. Jerison, and C. E. Kenig. Some new monotonicity theorems with applications to free boundary problems. Ann. of Math. (2), 155(2):369-404, 2002.

[5] L. A. Caffarelli, A. L. Karakhanyan, and F.-H. Lin. The geometry of solutions to a segregation problem for nondivergence systems. J. Fixed Point Theory Appl., 5(2):319-351, 2009.

[6] L. A. Caffarelli and F.-H. Lin. Singularly perturbed elliptic systems and multi-valued harmonic functions with free boundaries. J. Amer. Math. Soc., 21(3):847-862, 2008.

[7] L. A. Caffarelli, J.-M. Roquejoffre, and Y. Sire. Variational problems for free boundaries for the fractional Laplacian. J. Eur. Math. Soc. (JEMS), 12(5):1151-1179, 2010.

[8] L. A. Caffarelli and S. Salsa. A geometric approach to free boundary problems, volume 68 of Graduate Studies in Mathematics. American Mathematical Society, Providence, RI, 2005. 
[9] L. A. Caffarelli, S. Salsa, and L. Silvestre. Regularity estimates for the solution and the free boundary of the obstacle problem for the fractional Laplacian. Invent. Math., 171(2):425-461, 2008.

[10] L. A. Caffarelli and L. Silvestre. An extension problem related to the fractional Laplacian. Comm. Partial Differential Equations, 32(7-9):1245-1260, 2007.

[11] S.-M. Chang, C.-S. Lin, T.-C. Lin, and W.-W. Lin. Segregated nodal domains of twodimensional multispecies Bose-Einstein condensates. Phys. D, 196(3-4):341-361, 2004.

[12] M. Conti, S. Terracini, and G. Verzini. Nehari's problem and competing species systems. Ann. Inst. H. Poincaré Anal. Non Linéaire, 19(6):871-888, 2002.

[13] M. Conti, S. Terracini, and G. Verzini. An optimal partition problem related to nonlinear eigenvalues. J. Funct. Anal., 198(1):160-196, 2003.

[14] M. Conti, S. Terracini, and G. Verzini. Asymptotic estimates for the spatial segregation of competitive systems. Adv. Math., 195(2):524-560, 2005.

[15] M. Conti, S. Terracini, and G. Verzini. A variational problem for the spatial segregation of reaction-diffusion systems. Indiana Univ. Math. J., 54(3):779-815, 2005.

[16] E. N. Dancer, K. Wang, and Z. Zhang. Uniform Hölder estimate for singularly perturbed parabolic systems of Bose-Einstein condensates and competing species. J. Differential Equations, 251(10):2737-2769, 2011.

[17] E. N. Dancer, K. Wang, and Z. Zhang. The limit equation for the Gross-Pitaevskii equations and S. Terracini's conjecture. J. Funct. Anal., 262(3):1087-1131, 2012.

[18] A. de Pablo, F. Quirós, A. Rodríguez, and J. L. Vázquez. A general fractional porous medium equation. Comm. Pure Appl. Math., 65(9):1242-1284, 2012.

[19] S. Dipierro and A. Pinamonti. A geometric inequality and a symmetry result for elliptic systems involving the fractional Laplacian. J. Differential Equations, 255(1):85-119, 2013.

[20] M. M. Fall and V. Felli. Unique continuation property and local asymptotics of solutions to fractional elliptic equations. Comm. Partial Differential Equations. To appear.

[21] B. Noris, H. Tavares, S. Terracini, and G. Verzini. Uniform Hölder bounds for nonlinear Schrödinger systems with strong competition. Comm. Pure Appl. Math., 63(3):267-302, 2010.

[22] B. Noris, H. Tavares, S. Terracini, and G. Verzini. Convergence of minimax structures and continuation of critical points for singularly perturbed systems. J. Eur. Math. Soc. (JEMS), 14(4):1245-1273, 2012.

[23] B. Opic and A. Kufner. Hardy-type inequalities, volume 219 of Pitman Research Notes in Mathematics Series. Longman Scientific \& Technical, Harlow, 1990.

[24] L. Silvestre. Regularity of the obstacle problem for a fractional power of the Laplace operator. Comm. Pure Appl. Math., 60(1):67-112, 2007.

[25] H. Tavares and S. Terracini. Regularity of the nodal set of segregated critical configurations under a weak reflection law. Calc. Var. Partial Differential Equations, 45(3-4):273-317, 2012.

[26] S. Terracini, G. Verzini, and A. Zilio. Uniform Hölder bounds for strongly competing systems involving the square root of the laplacian. Preprint arXiv:1211.6087.

[27] S. Terracini, G. Verzini, and A. Zilio. Uniform Hölder regularity with small exponent in competition-fractional diffusion systems. Preprint arXiv:1303.6079.

[28] A. Zilio. On monotonicity formula, fractional operators and strong competition. PhD thesis, Politecnico di Milano, in preparation.

E-mail address: gianmaria.verzini@polimi.it

Dipartimento di Matematica, Politecnico di Milano, p.za Leonardo da Vinci 32, 20133 Milano, Italy

E-mail address: alessandro.zilio@polimi.it

Dipartimento di Matematica, Politecnico di Milano, P.za Leonardo da Vinci 32, 20133 Milano, Italy 